This item was submitted to Loughborough's Research Repository by the author.

Items in Figshare are protected by copyright, with all rights reserved, unless otherwise indicated.

\title{
Numerical simulation of mass transfer and fluid flow evolution of a rectangular free jet of air
}

PLEASE CITE THE PUBLISHED VERSION

https://doi.org/10.1016/j.ijheatmasstransfer.2017.10.030

PUBLISHER

(C) Elsevier

VERSION

AM (Accepted Manuscript)

\section{PUBLISHER STATEMENT}

This work is made available according to the conditions of the Creative Commons Attribution-NonCommercialNoDerivatives 4.0 International (CC BY-NC-ND 4.0) licence. Full details of this licence are available at: https://creativecommons.org/licenses/by-nc-nd/4.0/

\section{LICENCE}

CC BY-NC-ND 4.0

\section{REPOSITORY RECORD}

Di Venuta, Ivan, Ivano Petracci, Matteo Angelino, Andrea Boghi, and Fabio Gori. 2017. "Numerical Simulation of Mass Transfer and Fluid Flow Evolution of a Rectangular Free Jet of Air". figshare. https://hdl.handle.net/2134/26948. 


\title{
Numerical simulation of mass transfer and fluid flow evolution of a rectangular free jet of air
}

\author{
Ivan Di Venuta ${ }^{\mathrm{a}}$, Ivano Petracci ${ }^{\mathrm{a}}$, Matteo Angelino ${ }^{\mathrm{b}}$, Andrea Boghi ${ }^{\mathrm{c}}$, Fabio Gori ${ }^{\mathrm{a}, *}$ \\ a'Department of Industrial Engineering, University of Rome “Tor Vergata”, \\ Via del Politecnico 1, 00133 Rome, Italy. \\ boughborough University, Aeronautical and Automotive Engineering, \\ Stewart Miller Building, Loughborough, LE11 3TU, UK. \\ 'School of Water, Energy and Environment, Cranfield University, \\ Cranfield, Bedordshire, MK43 0AL, UK. \\ "Corresponding Author, gori@uniroma2.it
}

\begin{abstract}
The paper presents Large Eddy Simulations (LES) of mass transfer and fluid flow evolutions of a submerged rectangular free jet of air in the range of Reynolds numbers from $\operatorname{Re}=3400$ to $\operatorname{Re}=22,000$, with the Reynolds number, Re, defined with the hydraulic diameter of the rectangular slot, of height $\mathrm{H}$. The numerical simulations are $3 \mathrm{D}$ for $\mathrm{Re}=3400$ and 6800 , while $2 \mathrm{D}$ for $\mathrm{Re}=10,400$ and 22,000 to reduce computational time costs. The average and instant LES numerical simulations are compared with the concentration visualizations, obtained with the Particle Image Velocimetry (PIV) technique, and the fluid dynamics variables, velocity and turbulence, measured with the PIV technique and the Hot Film Anemometry (HFA). In the numerical simulations, the Schmidt number is equal to 100 to compare the air concentration in the PIV experiments, while the turbulence on the exit of the slot is equal to the value measured experimentally, and ranging between $1 \%$ and $2 \%$. The average 2-3D LES simulations are in agreement with the concentration and the fluid dynamics experimental results in the Undisturbed Region of Flow (URF) and in the Potential Core Region (PCR), while the vortex breakdown is captured only by the 3D LES approach. As far as the instant flow evolution is concerned, the 2-3D LES simulations reproduce the Negligible Disturbances Flow (NDF), where the jet height maintains constant, and the Small Disturbances Flow (SDF), where the jet height oscillates, with contractions and enlargements, but without the vortex formation. Average and instant velocity and turbulence numerical simulations on the centreline are in good agreement to the experimental PIV measurements.
\end{abstract}

Keywords: 2-3D Large Eddy Simulations; Transitional to turbulent flow; Numerical concentration and velocity fields compared to PIV visualizations; Numerical fluid dynamics variables compared to HFA measurements; Confirm of URF in average flow and NDF, SDF in instant flow.

\section{Nomenclature}

Latin

D diameter

E turbulent energy spectrum 
$H \quad$ height of the slot

$k \quad$ turbulent kinetic energy

$k_{B} \quad$ Boltzmann constant

l mean free path

$P \quad$ mean static pressure

$p \quad$ instantaneous static pressure

$r \quad$ particle radius

$S_{i j} \quad$ rate of shear tensor

$t \quad$ time

$U_{0} \quad$ average maximum velocity on the slot exit

$U_{\text {avg }}$ average velocity on the slot exit

$U_{\max } \quad$ average maximum velocity on centreline

$\boldsymbol{u}_{\boldsymbol{k}} \quad$ discrete perturbation velocity field

$U_{n} \quad$ complex Fourier modes

$x \quad$ axial coordinate

y cross-stream coordinate

Dimensionless parameters

$P e=R e \cdot S c \quad$ Peclet mass transfer number

$R e=\frac{U_{i n} D_{h}}{v} \quad$ Reynolds number

Sc $=\frac{v}{\Gamma} \quad$ Schmidt number

$S C_{T} \quad$ Turbulent Schmidt Number

$\mathrm{Tu} \quad$ Turbulence intensity

Greek

$\dot{\gamma} \quad$ shear rate

$\Gamma \quad$ concentration molecular diffusivity

$\Delta \quad$ filter width 
$v \quad$ kinematic viscosity

$v_{T} \quad$ turbulent viscosity

$\tau_{i j}^{R} \quad$ Reynolds stress tensor

\section{Subscripts}

sgs sub-grid scale

$T \quad$ turbulent

\section{Introduction}

The evolution of turbulent rectangular free jets has been widely investigated in the last decades from the experimental point of view, but at a lesser extent numerically. The flow evolution of a jet, emerging from a two-dimensional rectangular slot without converging duct, was investigated experimentally in [1]. The main conclusion was the interaction of the jet with the stagnant fluid just after the exit, defining two regions of flow. The first one is the potential core region, PCR, or zone of flow establishment, where the average velocity on the jet centerline remains equal to the exit one, while the second one, after the PCR, is the zone of established flow, or fully developed region, FDR. The origin of the FDR was identified by the distance of the hypothetical line-source from the slot exit. Average velocity measurements were performed in [2] with two types of rectangular slots founding two different lengths of the hypothetical line source, generally upstream the slot exit. As far as the distance of the line source is concerned, different lengths at the same Reynolds number were found in [3-4]. The deformation of a three-dimensional rectangular jet was investigated in [5] where the line-source of the jet was upstream the slot exit, independently from the Reynolds number.

The experiments carried out in [6-10], with average concentration visualizations and fluid dynamics measurements, brought to the conclusion that velocity and turbulence remain unchanged, compared to those measured on the exit, for a length decreasing with the increasing Reynolds number. This type of average flow was called Undisturbed Region of Flow, URF, and the presence of an impinged body, as a cylinder, reduces its length, [11]. The experiments with instant concentration visualizations of the flow evolution, [12], showed that the URF can be further subdivided into two types of flow. The first one is the negligible disturbances flow, NDF, where the jet height maintains constant, while the second one is the small disturbances flow, SDF, where the jet height oscillates, with contractions and/or enlargements, without the formation of vortex. The region of coherent vortices, present after the SDF, precedes the vortex breakdown.

As far as the theoretical approach to the jet flow evolution is concerned, Tollmien [13] and Görtler [14] studied the flow of turbulent rectangular submerged free jets, and proposed a self-similar evolution for 
the axial velocity in the potential core region, PCR, and the fully developed region, FDR. The equations describing the velocity trend have been confirmed experimentally in [15-18] for the PCR, and in [19-21] for the FDR.

The new flow evolution of the jet can be important in several heat transfer applications: as a jet impinging a single smooth cylinder, [22-28], a finned cylinder, [29-33]; two cylinders in a row, [34-35]; and three cylinders in a row, [36-37]. The numerical results of [38-42] confirmed that the URF, as well as the NDF and SDF, are predictable numerically.

The aim of the present paper is to perform 2-3 D Large Eddy Simulations (LES) at four Reynolds numbers in the range from 3400 to 22,000, to compare the numerical simulations with the concentration and velocity visualizations of the Particle Image Velocimetry (PIV), and the fluid dynamic measurements of the Hot Film Anemometry (HFA).

\section{Numerical Method}

\section{Governing Equations}

The Large Eddy Simulation (LES) approach allows to solve the large-scale turbulent structure and to model small-scale ones through a spatial filtering of the Navier-Stokes equations, leading to a reduction of the computational costs, but allowing to capture the fluid dynamics. If $a\left(t, x_{i}\right)$ is a generic field, function of the time $t$ and the spatial coordinate, $X_{i}$, it is possible to define a grid-scale filtered field $\tilde{a}\left(t, x_{i}\right)$

$$
\tilde{a}\left(t, x_{i}\right)=\int_{\Omega} a\left(t, \xi_{i}\right) g\left(x_{i}-\xi_{i}, \Delta\right) d^{3} \xi_{i}
$$

where $\Omega$ is the domain extension and $g$ the spatial filter, function of the width $\Delta$. The application of the filtering approach to the conservation equations, expressed in non-dimensional form, allows obtaining the following equations:

mass conservation

$$
\frac{\partial \tilde{u}_{i}}{\partial x_{i}}=0
$$

momentum conservation

$$
\frac{\partial \tilde{u}_{i}}{\partial t}+\frac{\partial}{\partial x_{j}}\left(\tilde{u}_{i} \tilde{u}_{j}+\tilde{p} \delta_{i j}-\frac{2}{\operatorname{Re}} \tilde{S}_{i j}\right)=\frac{\partial \tau_{i j}^{s g s}}{\partial x_{j}}
$$

concentration conservation 


$$
\frac{\partial \tilde{c}}{\partial t}+\frac{\partial}{\partial x_{k}}\left(\tilde{c} \cdot \tilde{u}_{k}-\frac{1}{\operatorname{Re}} \frac{1}{S c} \frac{\partial \tilde{c}}{\partial x_{k}}\right)=-\frac{\partial J_{k}^{s g s}}{\partial x_{k}}
$$

being $S_{i j}$ the rate of shear tensor, defined as

$$
\tilde{S}_{i j}=\frac{1}{2}\left(\frac{\partial \tilde{u}_{i}}{\partial x_{j}}+\frac{\partial \tilde{u}_{j}}{\partial x_{i}}\right)-\frac{1}{3} \frac{\partial \tilde{u}_{k}}{\partial x_{k}} \delta_{i j}
$$

where $u_{i}$ is the velocity vector, $p$ the static pressure, $\delta_{i j}$ the identity tensor, $C$ the concentration.

The Reynolds number is defined with the inlet velocity, $U_{i n}$, the hydraulic diameter, $D_{h}$, and the kinematic viscosity, $\boldsymbol{v}$,

$$
\operatorname{Re}=\frac{U_{i n} D_{h}}{v}
$$

while the Schmidt number requires also the concentration molecular diffusivity $\Gamma$

$$
S c=\frac{v}{\Gamma}
$$

The diffusivity, $\Gamma$, of a particle, taken into account by the Einstein-Smoluchowski diffusion equation [43], can be evaluated with the Cunningham empirical equation [44],

$$
\Gamma=\left(1+\frac{l}{r}\left(1.257+0.4 \exp \left(-\frac{1.1 \cdot r}{l}\right)\right)\right) \frac{k_{B} \cdot T}{6 \cdot \pi \cdot \mu \cdot r}
$$

where $r$ is the particle radius and $l$ the mean free path of the molecules of air. Since the typical values of the particle radius in PIV applications is in the range, $r \approx 10^{-6}-10^{-5} \mathrm{~m}$, the Schmidt number is in the range Sc $\in[10,100]$.

The sub-grid stress tensor, $\tau_{i j}^{\text {sgs }}$, is modeled with the “diffusive gradient” hypothesis

$$
\tau_{i j}^{s g s}=2 v_{s g s} S_{i j}-\frac{2}{3} k_{s g s} \delta_{i j}
$$

Similarly, the sub-grid mass flux vector, $J_{k}^{s g s}$, is

$$
J_{k}^{s g s}=-\frac{v_{s g s}}{S c_{s g s}} \frac{\partial \tilde{c}}{\partial x_{k}}
$$


where the sub-grid Schmidt number, $S c_{s g s}$, and the sub-grid viscosity, $v_{s g s}$, are

$v_{s g s}=C_{S} \Delta^{2} \tilde{S}$

The sub-grid kinetic energy, $k_{\text {sgs }}$,

$k_{s g s}=C_{I} \Delta^{2} \tilde{S}^{2}$

the filtered shear rate, $\tilde{S}$,

$\tilde{S}=\sqrt{2 \tilde{S}_{i j} \tilde{S}_{i j}}$

and the filter width, $\Delta$, are

$\Delta=\sqrt{\Delta_{k} \Delta_{k}}$

where $\Delta_{k}$ is the box filter width in the k-th direction. The dynamic Smagorinsky model, developed in [45], is employed.

\section{Computational details}

The LES simulations are carried on with the finite-volume solver, pisoFoamPS, developed and implemented in the open-source code, OpenFOAM, to solve the Navier-Stokes equations, and the concentration one. The pisoFoamPS is a transient solver for incompressible turbulent flow, which uses the PISO algorithm, where the sub-grid Schmidt number is equal to 1 . The computational grids are generated with blockMesh, the OpenFOAM utility for mesh generation. The principle behind blockMesh is to decompose the domain geometry into a set of hexahedral blocks to get a structured grid. As far as the 3D approach is concerned, the geometry is made of a rectangular box, 3 slot wide in the transverse direction (z), 6 slot height in the vertical one $(y)$ and 13 slot long in the axial one $(x)$. The grid is uniform in the $z$ direction, and stretched towards the slot exit in the $y$ and $x$ directions near the slot exit, although the minimum and maximum grid stencils are of the same order of magnitude. In the $z$ direction the stencil is $\Delta z=0.0387$ slot height, while in the $x$ and $y$ directions they are $\Delta x, \Delta y=0.0148 \div 0.0588$ slot height. The grid is made of $442 \times 262 \times 76$ points. The 2D geometry is the same in the $x-y$ plane, but grids with the stencil decreasing for the increasing Re are used in order to have $\left(S c / S c_{\text {sgs }}\right) \Delta^{2} \operatorname{Re} \cdot \max \left(C_{S} \tilde{S}\right)<0.1$ for all cases, to insure that the sub-grid effects do not affect the solution at the jet boundaries. The dimensions of the 2D computational grids are listed in Table 1 . The "simple" one filter, chosen for the LES simulation with the 
dynamic Smagorinsky model, interpolates the field in a given point with its neighboring cells. The filter amplitude is equal to the cubic root of the cell volume.

Table 1- 2D Computational grids

\begin{tabular}{|l|l|l|l|}
\hline Reynolds number & Peclet number & x-direction nodes & y-direction nodes \\
\hline 10,400 & $1.04 \cdot 10^{6}$ & 884 & 524 \\
\hline 22,000 & $2.2 \cdot 10^{6}$ & 884 & 524 \\
\hline
\end{tabular}

The turbulent velocity profile imposed on the slot exit to reproduce the experimental conditions of $[10,12]$ is given by

$$
U(y)=U_{o} \tanh \left(\frac{H}{\theta}\left(1-\left|\frac{y}{H / 2}\right|\right)\right)
$$

where $\theta$ is the momentum thickness, approximated by the relation

$$
\theta=\frac{H}{\ln (2)}\left(1-\frac{U_{a v}}{U_{0}}\right)
$$

with $U_{0}$ the maximum velocity on the slot exit and $U_{\text {avg }}$ the average velocity. The wall above and below the slot has a thickness of 0.176 hydraulic diameters, while everywhere, in the $\mathrm{x}$ and $\mathrm{y}$ direction, there is a free boundary.

The remaining patches of the domain are the outlets, where the total pressure and the velocity on the boundaries, known in OpenFOAM as "pressureInletOutletVelocity", are specified. The characteristic of change, according to the velocity direction, are assumed in order to avoid continuity errors. If the fluid flows out of the domain a zero gradient condition is imposed for the velocity, otherwise the velocity is derived from the flux in the patch-normal direction. As far as the concentration is concerned, a flat profile is imposed on the inlet and a Neumann boundary condition everywhere else. As far as the time and spatial discretization are concerned, a second order backwards is chosen for the explicit time integration scheme and the time step in each simulation satisfies the condition CFL $<0.5$, while the numerical schemes is a second order central for the spatial derivatives.

The transient state lasts about 10 "flow time", which is the ratio between the domain length and the axial velocity on the slot exit. After 10 flow times several probes are placed in the computational domain to analyze the average values of velocity, vorticity and concentration, which are collected as average when those fields do not change over time. In conclusion, 27 flow times are necessary to obtain the average variables in the 3D numerical simulations, while 20 flow times are enough in the 2D cases. 
Some disturbances are added to the velocity profile in order to trigger the turbulence in the simulations. A small perturbation of few percent in the amplitude of the velocity profile is enough to produce a 3D motion, even with 2D boundary conditions. The technique proposed in [46] is adopted, with the advantage of injecting the disturbance in the low and intermediate wave number ranges, where it cannot be immediately damped.

Let decompose the discrete perturbation velocity field $\boldsymbol{u}_{\boldsymbol{k}}$ in Fourier series

$$
u_{k}=\frac{1}{N} \sum_{n=0}^{N-1} U_{n} e^{2 \pi i k n / N}
$$

where $U_{n}$ are the complex Fourier modes, which can be written as $U_{n}=\left|U_{n}\right| e^{i \varphi_{n}}$. According to the Parseval theorem

$$
\sum_{n=0}^{N-1} E_{n}=\sum_{m=0}^{N-1}\left|u_{m}\right|^{2}=\frac{1}{N} \sum_{n=0}^{N-1}\left|U_{n}\right|^{2}
$$

In order to generate a perturbation, able to reproduce the turbulent energy spectrum, it is assumed that $\left|U_{n}\right|=\sqrt{N E_{n}}$, with $\varphi_{n}$ a random function of $\mathrm{n}$.

The turbulent spectrum is given by

$$
E(k) \propto k^{4} e^{-2\left(\frac{k}{k_{0}}\right)^{2}}
$$

with

$$
k=\sqrt{k_{x}^{2}+k_{y}^{2}+k_{z}^{2}}
$$

The inlet perturbation field is assumed to act in the three directions, being function of both vertical and spanwise coordinates.

\section{Numerical results}

The numerical simulations are carried on at four Reynolds numbers, i.e. 3400, 6800, 10,400 and 22,000. The numerical simulations are 3D for the two smallest Reynolds numbers, while 2D at the two greatest ones, to save computational time costs. In the numerical simulations, as far as the concentration is concerned, the Schmidt number is equal to 100 , in order to compare the numerical simulations with the PIV concentration 
visualizations, because the numerical concentration fields are very similar for $\mathrm{Sc}=10$. The turbulence assumed on the exit of the slot is equal to $1.5-2 \%$, as measured experimentally in $[10,12]$.

$3.1-3 D, R e=3400$.

Figure 1 presents the average concentration field (colored), obtained with the 3D numerical simulation at $\mathrm{Re}=3400$. The height of the jet remains constant, and equal to the slot one, for a length equal to $\mathrm{x} / \mathrm{H}=3-4$, which identifies the length of the URF in the average numerical simulations. After the URF the darker concentration field decreases in thickness, identifying visually the Potential Core region, PCR, which ends around $\mathrm{x} / \mathrm{H}=7$. Both the lengths of URF and PCR agree with the experimental results of [10].

The numerical results of the average concentration field are compared to the PIV average visualization, obtained for the same Reynolds number in [10], and reported on the same Fig. 1 with its envelope as dotted line. It is evident the good agreement between the 3D numerical simulations and the PIV average visualization envelope.

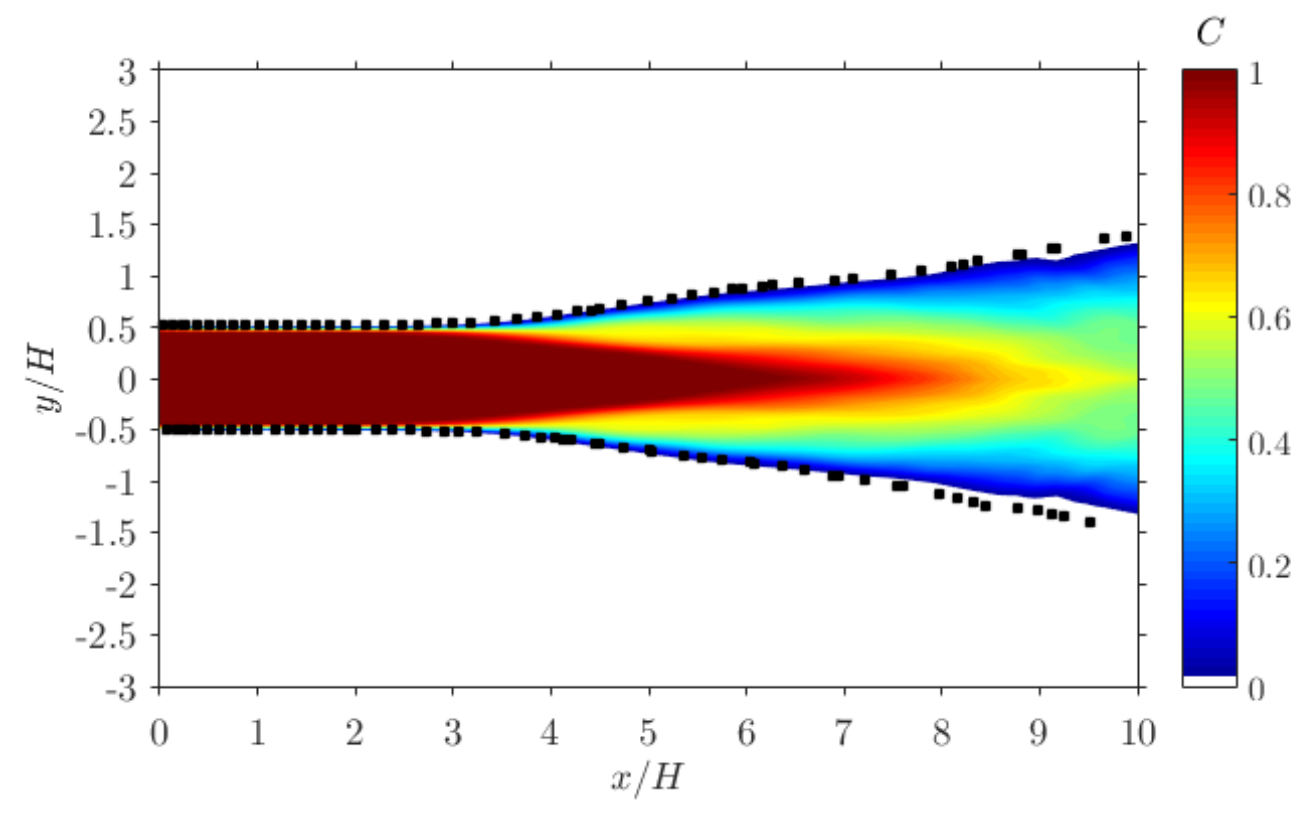

Fig. 1 - Average concentration field: 3D numerical (colored) and PIV envelope (dashed line) at $R e=3400$.

The average velocity field, obtained with the 3D numerical simulation at $\operatorname{Re}=3400$, is shown in Fig. 2a. The height of the jet remains almost constant for a length equal to $\mathrm{x} / \mathrm{H}=4$, which identifies the length of the URF in the average numerical simulations. The darker triangular region, present after the URF and identifying visually the Potential Core Region, PCR, ends around $\mathrm{x} / \mathrm{H}=7$. Both the lengths of URF and PCR agree with the experimental PIV results of [10]. The 3D numerical results of the average velocity can be compared to the PIV measurements, obtained for the same Reynolds number, [10], and reported in Fig. 2b. It is evident the good agreement between the 3D numerical simulations and the PIV results. 


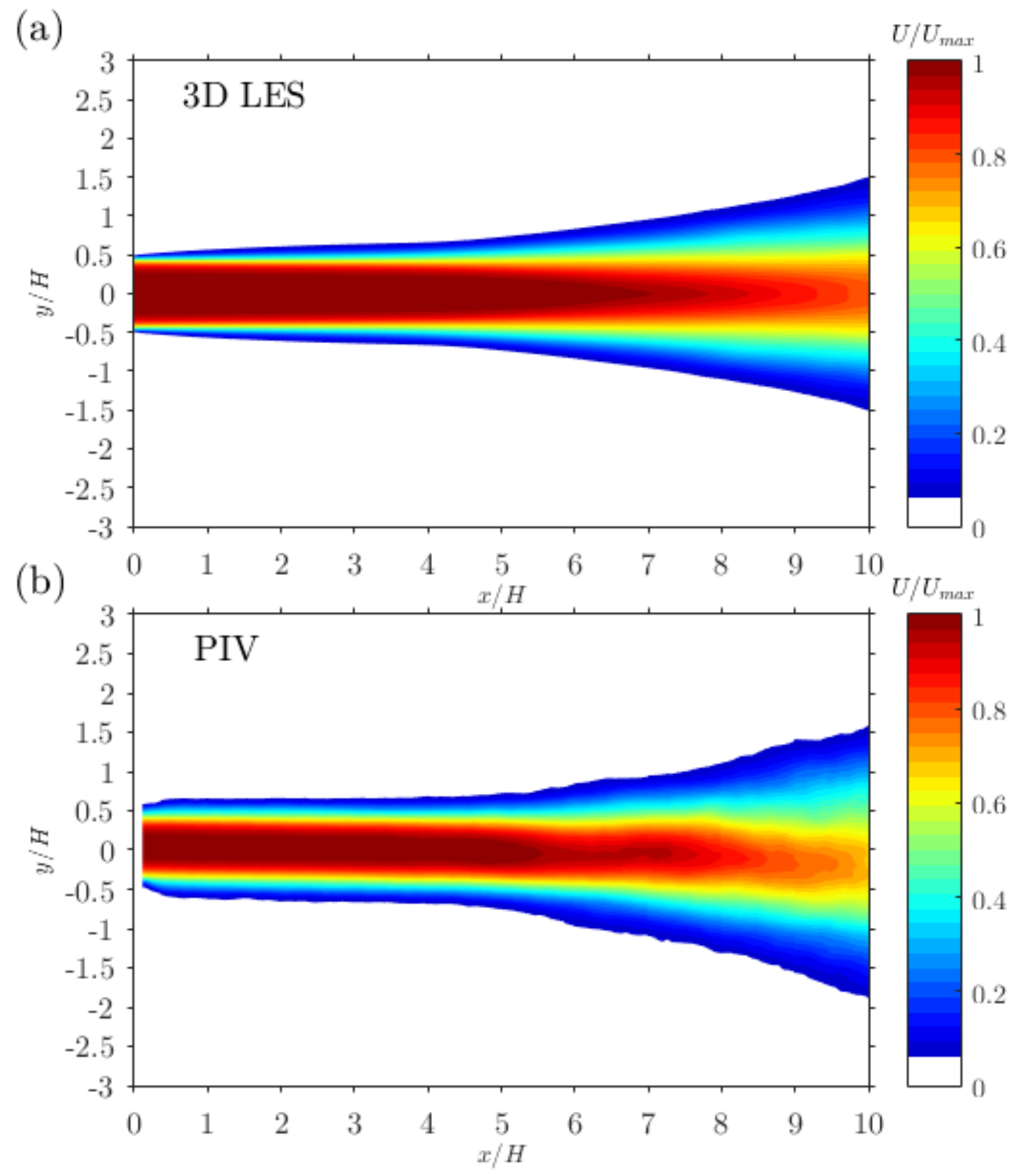

Fig. 2 - Average velocity field: 3D numerical (a) and experimental PIV (b) results at Re=3400.

The average velocity profiles, predicted numerically from the slot exit up the end of the PCR at $\mathrm{Re}=3400$, and presented in Fig. 3 with dots, are in good agreement to the average velocity measurements, [10], reported with circles. The average turbulent velocity profile on the exit of the slot, given by Eqs. 15-16, is reported in Fig. 3a with a continuous line, in agreement to the experimental turbulent velocity profile measured in the experiments [10]. 

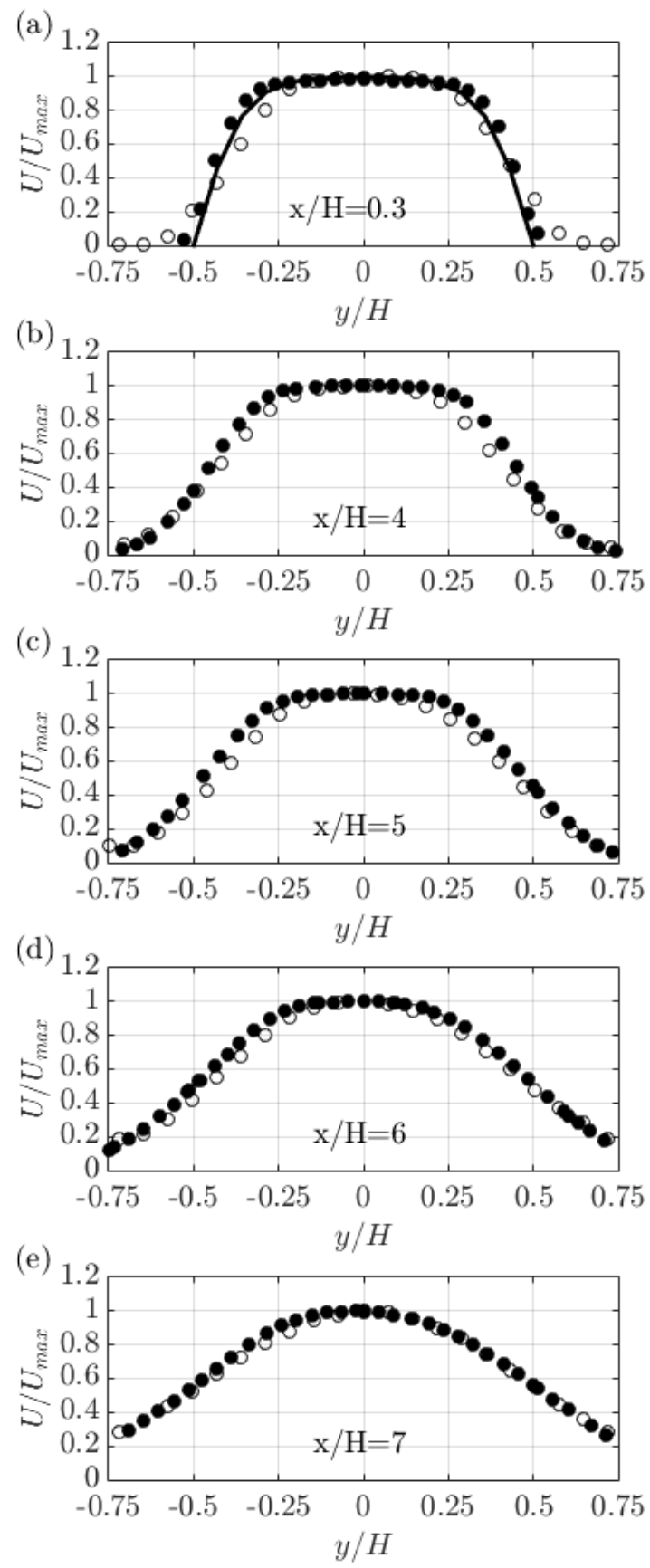

Figure 3 - Average velocity profiles: numerical (•) and PIV measurements ( $)^{)}$at $R e=3,400$.

The instant concentration fields, obtained with the 3D numerical simulations at $\mathrm{Re}=3400$, are reported, colored, in Figs. 4 (a, b, c) at three different instants. They are in qualitative agreement to the three experimental instant concentration PIV images, black and white, identified by the frames 13, 14, 43, obtained in the experiments [12] and never published before.

Figure 4a shows the negligible disturbances flow, NDF, long about $\mathrm{x} / \mathrm{H}=3$, and followed by the small disturbances flow, SDF, up to $\mathrm{x} / \mathrm{H}=4.5$, in good agreement to the experimental lengths, [12]. In the SDF the height of the jet increases, not symmetrically, without the formation of vortices. After the SDF, two pairs of 
vortices appear before the vortex breakdown. The vortices are not symmetric, as the PIV image of frame 13, Fig. 4d, typical of a laminar-transitional flow, with a qualitative good agreement. Figure 4b shows a shorter $\mathrm{NDF}$, long about $\mathrm{x} / \mathrm{H}=2-3$, followed by the $\mathrm{SDF}$, up to $\mathrm{x} / \mathrm{H}=3.5$, and by three couple of vortices, here almost symmetric, and by the vortex breakdown, after $\mathrm{x} / \mathrm{H}=9$. Figure $4 \mathrm{e}$, with the frame 14 , shows a similar instant PIV image. Figure 4c reports an image of the jet where the NDF is longer than in the previous figures, about $\mathrm{x} / \mathrm{H}=3.5$, and is followed by the SDF, with a slight contraction of the height, and two couple of vortices, in good agreement to the PIV image of the frame 43, Fig. 4f.

(a)

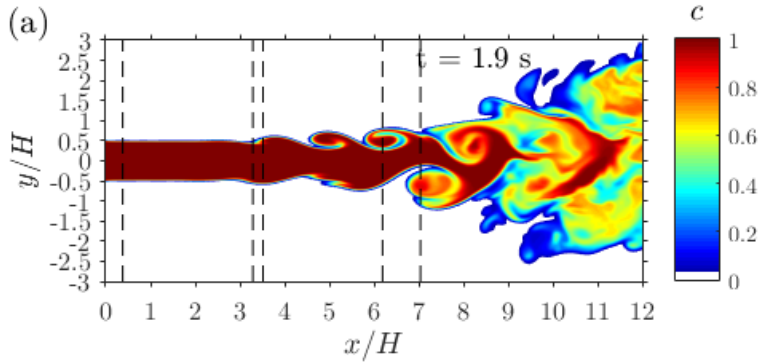

(b)

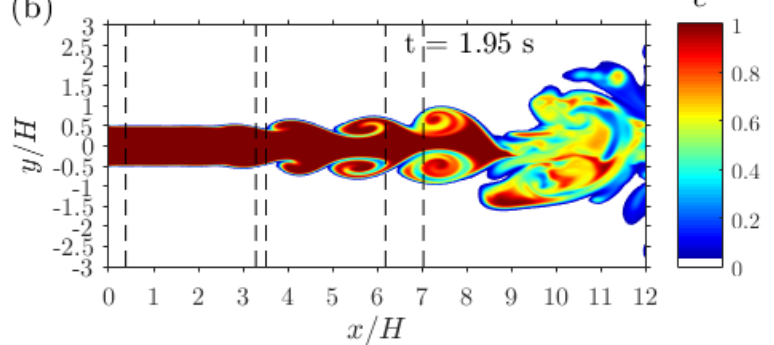

(c)

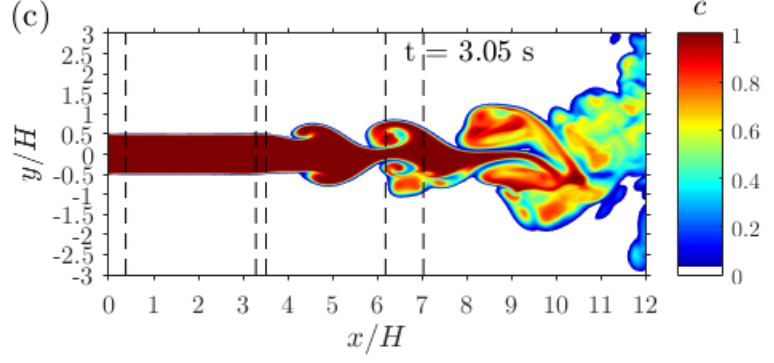

(d)

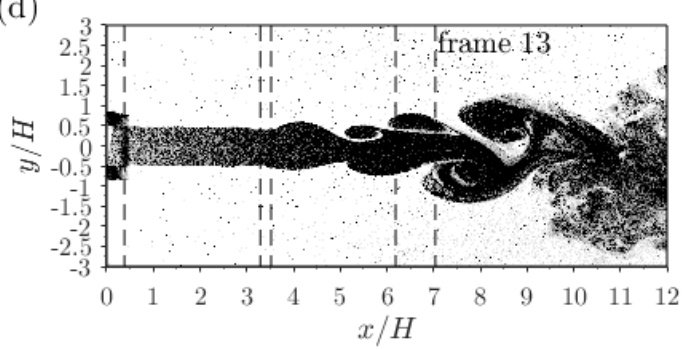

(e)

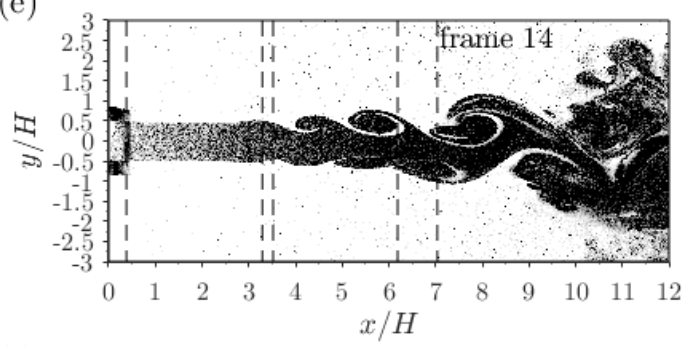

(f)

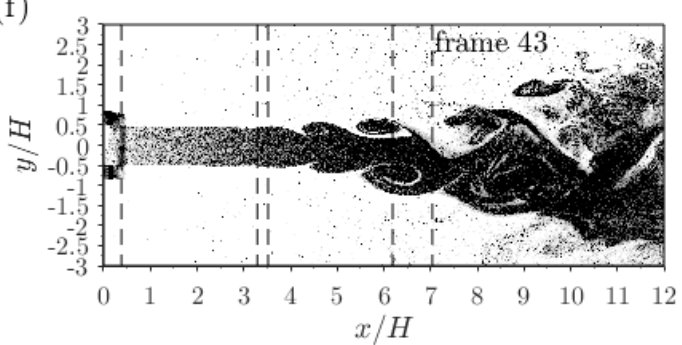

Fig. 4 - Instant concentrations: 3D numerical (colored, a,b,c) and experimental PIV (black-white, d,e,f) results at $\boldsymbol{R e}=\mathbf{3 4 0 0}$.

Figure 5 presents the numerical velocity and turbulence intensity results on the centreline (colored), average and instant, as Fig. $5 \mathrm{a}$ and $5 \mathrm{~b}$, at the times $1.95 \mathrm{~s}$ and $3.05 \mathrm{~s}$, and corresponding to the Figs. 4b and 4c. Figure 5 reports the experimental measurements of [12], as Fig. 5c and 5d, corresponding to the frames 14 and 43 of Figs. 4e and 4f. The numerical simulations appear in qualitative good agreement to the experimental measurements. The LES profiles appear smoother, compared to the PIV ones, because the velocity field in the LES are "filtered", while the PIV are experimentally captured at all the frequencies. 
(a)

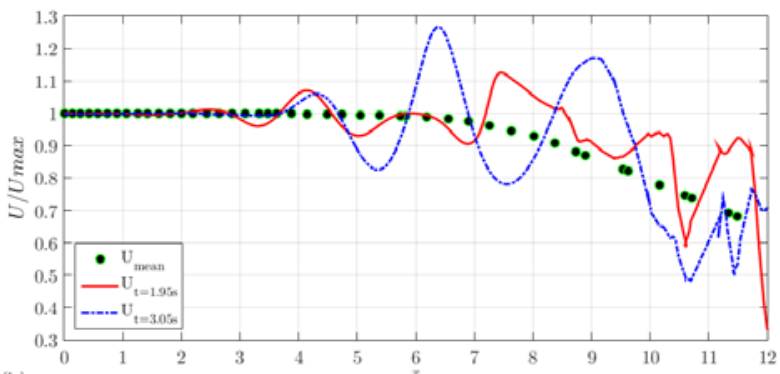

(b)

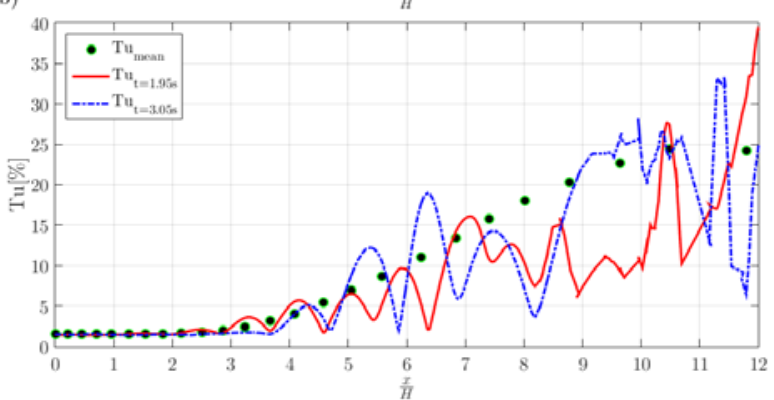

(c)

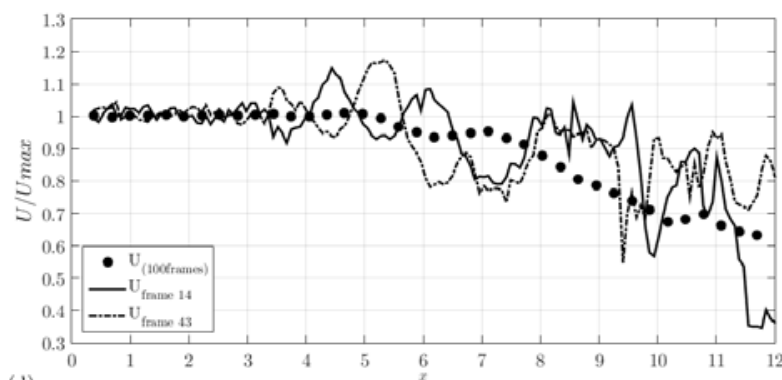

(d)

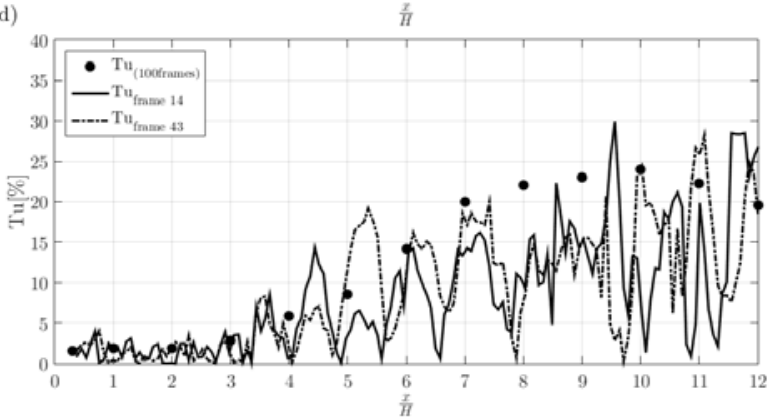

Fig.5 - Average and instant velocity and turbulence on the centreline: numerical (colored, a,b) and experimental results (black-white, $c, d)$ for $\operatorname{Re}=3400$.

The instant velocity profiles, predicted numerically at several distances from the slot exit up to the end of the PCR, are reported in Fig. 6 with circle and stars, respectively after $t=1.95 \mathrm{~s}$ and $\mathrm{t}=3.05 \mathrm{~s}$, and compared to the instant measurements of [12], reported with dotted and continuous lines, and relative to the frames 14 and 43. The distances from the slot exit where the comparisons are carried on in Fig. 6 are also indicated in Fig. 4 with vertical dotted lines.

On the exit of the jet, $\mathrm{x} / \mathrm{H}=0.38$, Fig. $6 \mathrm{a}$, the two numerical predictions are in very good agreement with the measurements, as well as at $\mathrm{x} / \mathrm{H}=3.29$, Fig. $6 \mathrm{~b}$, where the flow is still within the NDF. At the distance of $\mathrm{x} / \mathrm{H}=3.52$, Fig. $6 \mathrm{c}$, the velocity around the centerline, shown at $\mathrm{t}=1.95 \mathrm{~s}$, frame 14 , is greater than at $t=3.05 \mathrm{~s}$, frame 43 , because of the jet contraction, shown by Fig. $4 \mathrm{~b}$ and $4 \mathrm{e}$, frame 14 , in comparison to the jet height of Fig. 4c and 4f, frame 43. In conclusion, the jet flow is in the SDF, where a contraction of the jet height produces a velocity increase.

At the distance of $\mathrm{x} / \mathrm{H}=6.19$, Fig. $6 \mathrm{~d}$, the flow is in the coherent vortices region. The velocity around the centerline is smaller at $\mathrm{t}=1.95 \mathrm{~s}$, frame 14 , than at $\mathrm{t}=3.05$, frame 43 , because the jet height has an enlargement, evidenced by Fig. 4b and 4e, frame 14, in contrast to the contraction shown by Fig. 4c and 4f, frame 43. At the distance of $\mathrm{x} / \mathrm{H}=7.04$, Fig. 6e, the velocity around the centerline is smaller at $\mathrm{t}=3.05 \mathrm{~s}$, frame 43, than at $\mathrm{t}=1.95$, frame 14, because the jet height has an enlargement, evidenced by Fig. $4 \mathrm{c}$ and $3 \mathrm{f}$, frame 43, in contrast to the contraction shown in Fig. $4 \mathrm{~b}$ and 4e, frame 14. 

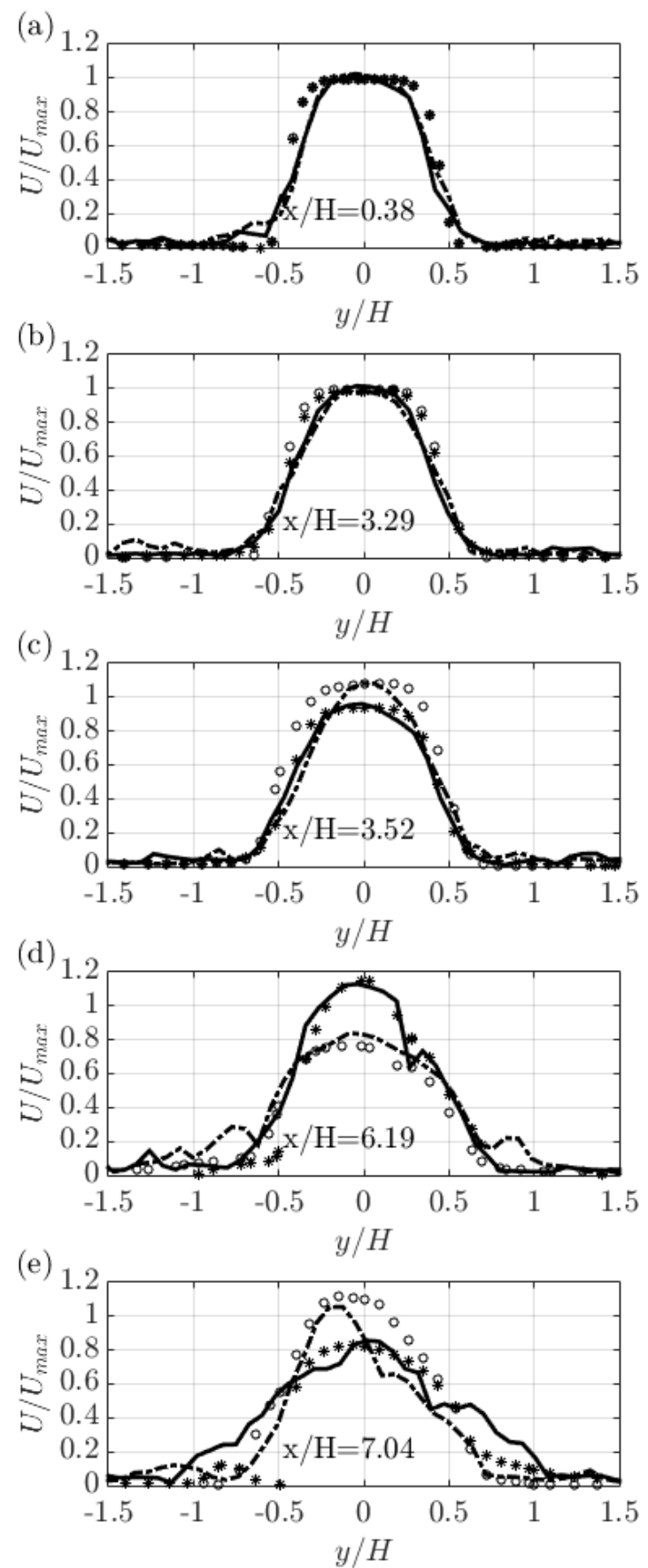

Figure 6 - Instant velocity profiles at $R e=3,400$. Numerical ( $\circ t=1.95 \mathrm{~s}, * t=3.05 \mathrm{~s}$ ) and PIV measurements (dotted line, frame 14; continuous line, frame 43).

$3.2-3 D, R e=6800$.

Figure 7 presents the average concentration field (colored), obtained with the 3D numerical simulation at $\mathrm{Re}=6800$. The height of the jet remains constant, and equal to the slot one, for a length equal to $\mathrm{x} / \mathrm{H}=3$, which identifies the length of the URF in the average numerical simulations. After the URF the 
darker concentration field decreases in thickness, identifying visually the Potential Core Region, PCR, which ends around $\mathrm{x} / \mathrm{H}=6-7$. Both the lengths of URF and PCR agree with the experimental results of [10].

The numerical results of the concentration field are compared to the PIV average visualization, obtained for the same Reynolds number in [10], and reported on the same Fig. 7 with its envelope as dotted line. It is evident the good agreement between the 3D numerical simulations and the PIV average visualization envelope.

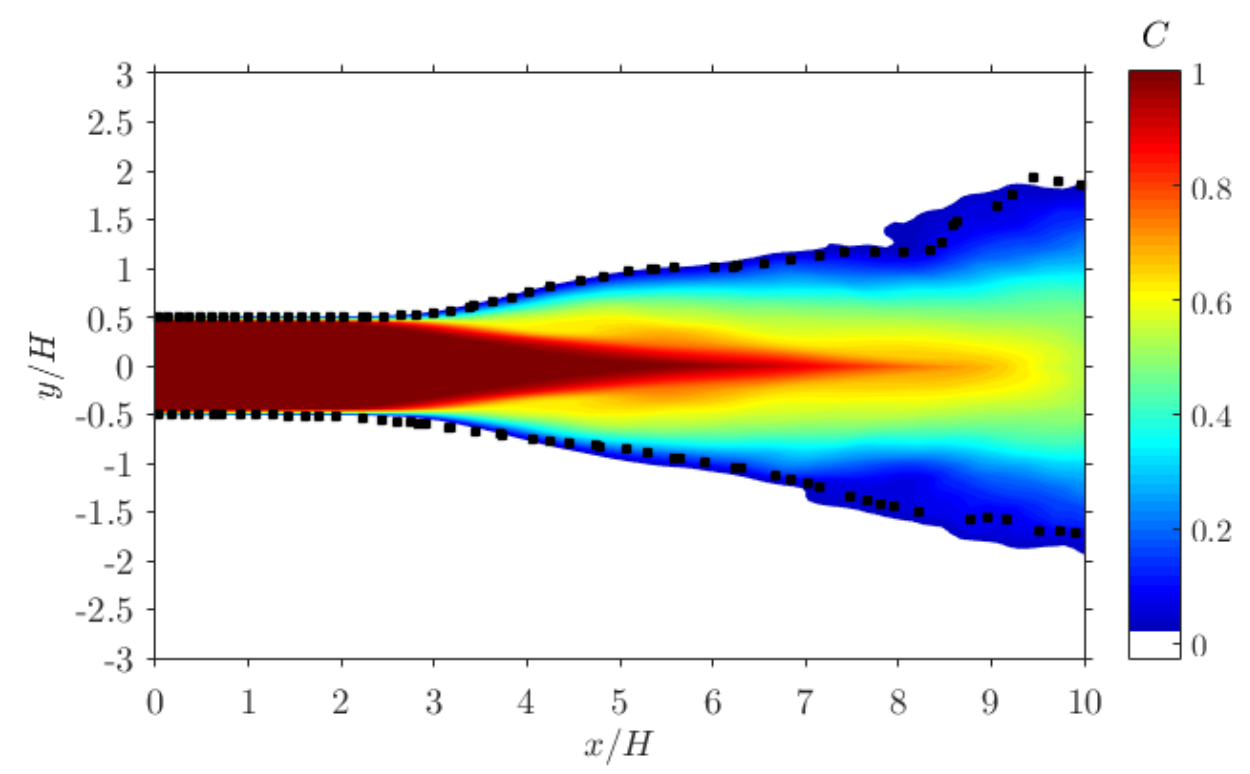

Fig. 7 - Average concentration field: 3D numerical (colored) and PIV envelope (dashed line) for Re=6800.

The average velocity field at $\mathrm{Re}=6800$, obtained with the 3D numerical simulation, is shown in Fig. 8a. The height of the jet remains almost constant for a length equal to about $\mathrm{x} / \mathrm{H}=3$, which identifies the length of the URF in the average numerical simulations. The darker triangular region, present after the URF and identifying visually the Potential Core region, PCR, ends around $x / H=6-7$. Both the lengths of URF and PCR agree with the experimental results of [10]. The 3D numerical results of the average velocity can be compared to the PIV measurements, obtained for the same Reynolds number, [10], and reported in Fig. 8b. Also for this Reynolds number it is evident the good agreement between the 3D numerical simulations and the PIV results. 
(a)

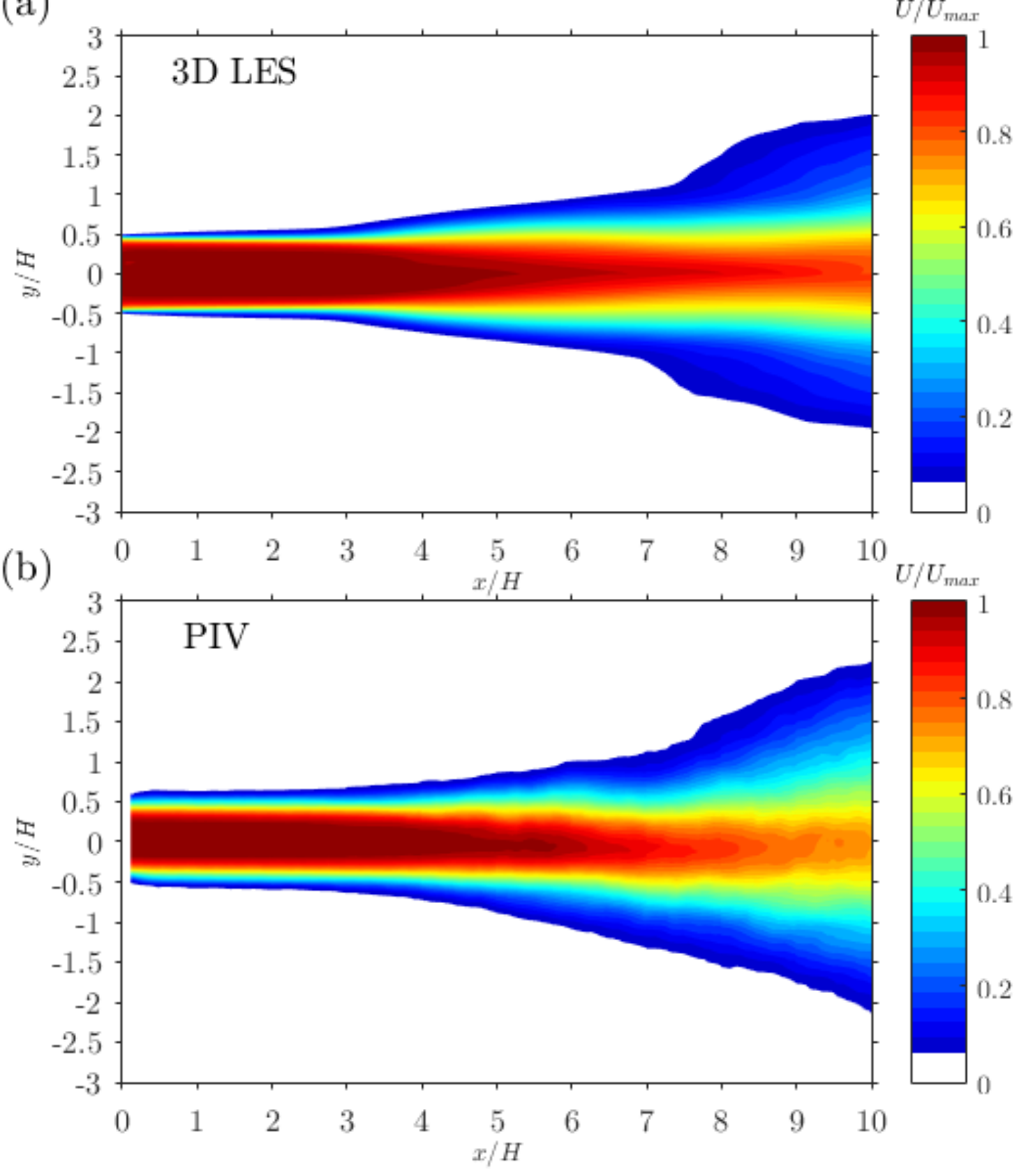

Fig. 8 - Average velocity field: 3D numerical (a) and experimental PIV (b) results at Re=6800.

The average velocity profiles, predicted numerically from the slot exit up the end of the PCR at $\mathrm{Re}=6800$, and presented in Fig. 9 with dots, are in reasonable good agreement to the average velocity measurements, [10], reported with circles. The average turbulent velocity profile on the exit of the slot, given by Eqs. 15-16, is reported in Fig. 9a with a continuous line, in agreement to the experimental turbulent velocity profile measured in the experiments [10]. 

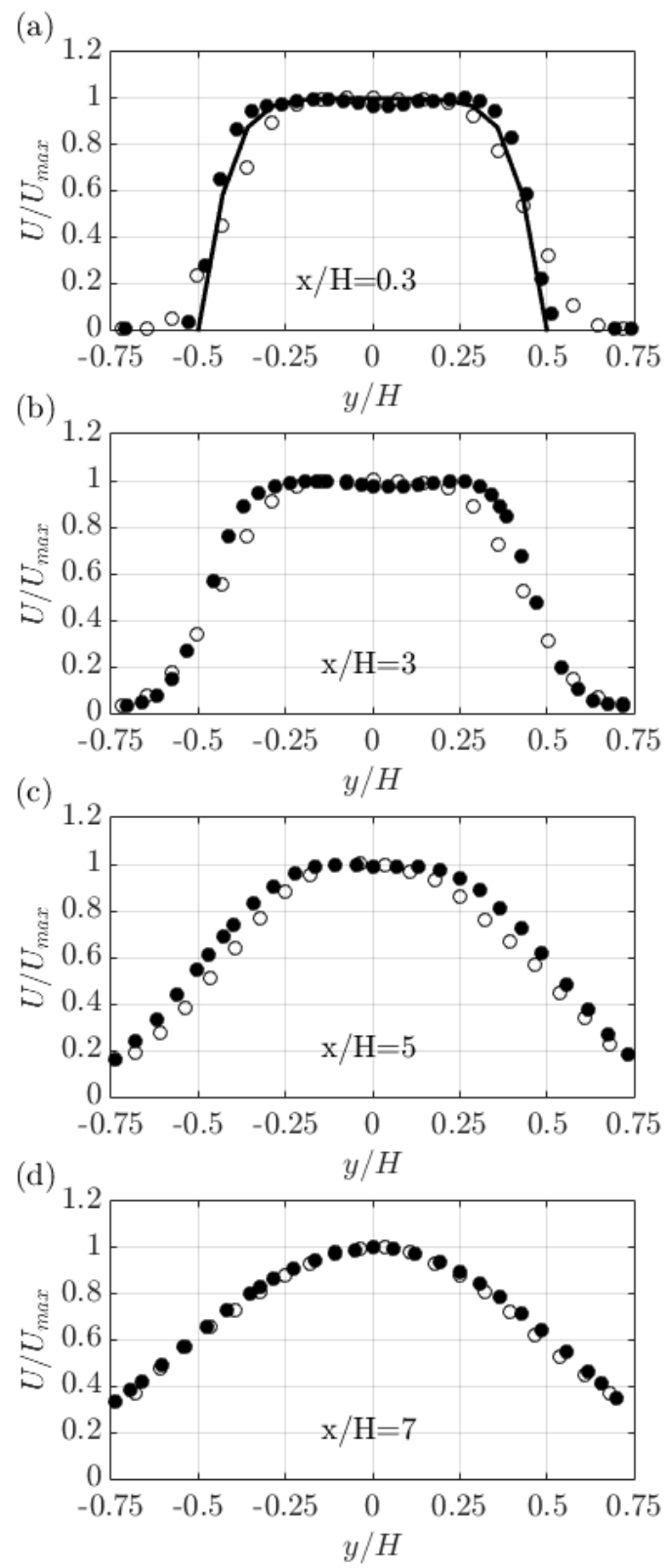

Figure 9 - Average velocity profiles: numerical (•) and PIV measurements (०) at $\operatorname{Re}=6800$.

The instant concentration fields at $\mathrm{Re}=6800$, obtained with the 3D numerical simulations, are reported, colored, in Figs. 10 (a, b) at two different instants. They are in qualitative agreement to the experimental instant concentration PIV images, black and white, and identified by the frames 33, 80, obtained in the experiments [12] and never published before.

Figure 10a shows the negligible disturbances flow, NDF, long about $\mathrm{x} / \mathrm{H}=2-3$, and followed by the small disturbances flow, $\mathrm{SDF}$, up to $\mathrm{x} / \mathrm{H}=4$, in good agreement to the experimental lengths [12]. After the SDF, the two pairs of vortices, present before the vortex breakdown, are symmetric, as those of the frame 33 in Fig. 10c, because the flow is turbulent. The PCR is ending after the second pair of vortices, around $\mathrm{x} / \mathrm{H}=7$ 8 , with an evident good agreement with the frame 33. Figure 10b reports the numerical concentration field 
after $\mathrm{t}=2.32 \mathrm{~s}$ with the $\mathrm{NDF}$, up to $\mathrm{x} / \mathrm{H}=3$, followed by the $\mathrm{SDF}$, with a contraction, up to $\mathrm{x} / \mathrm{H}=3.5$, and by two pairs of symmetric vortices and the vortex breakdown, after $\mathrm{x} / \mathrm{H}=6-7$. Figure 10d shows the PIV image, frame 80 , which is very similar to the numerical prediction, as far as NDF, SDF, the two pairs of symmetric vortices and the vortex breakdown are concerned.

(a)

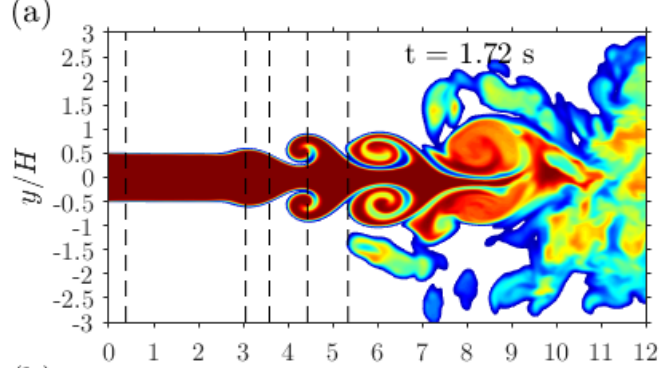

(b)

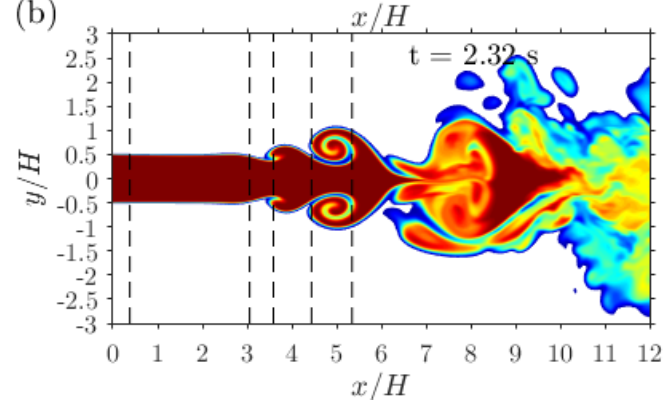

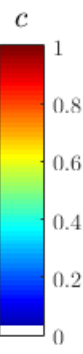

(c)

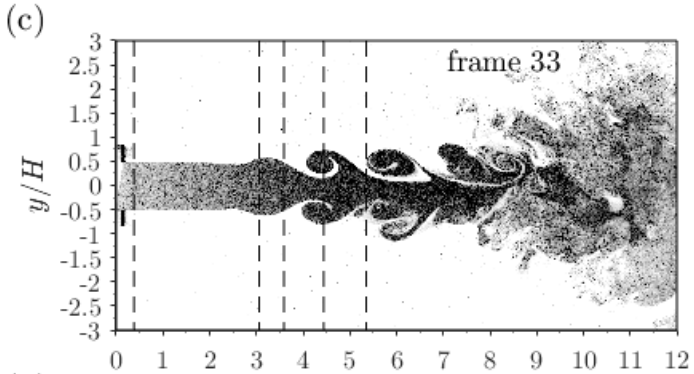

(d)

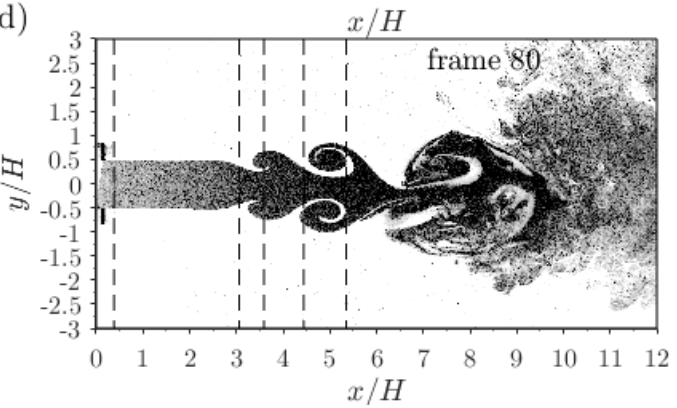

Fig. 10 - Instant concentration field: numerical (colored, $a, b)$ and experimental PIV (black-white, c,d) results at $\operatorname{Re}=6800$.

Figure 11 presents the average and instantaneous velocity and turbulence intensity numerical results on the centreline at the times $\mathrm{t}=1.72 \mathrm{~s}$ and $\mathrm{t}=2.32 \mathrm{~s}$, the same of Fig. 10a and 10b. Figure 11 reports also the corresponding experimental measurements, [12], by the frames 33 and 80, as Fig. 11c and 11d. The numerical simulations appear in qualitative good agreement to the experimental measurements. Once again, the numerical results appear smoother because of the filtered velocity. 

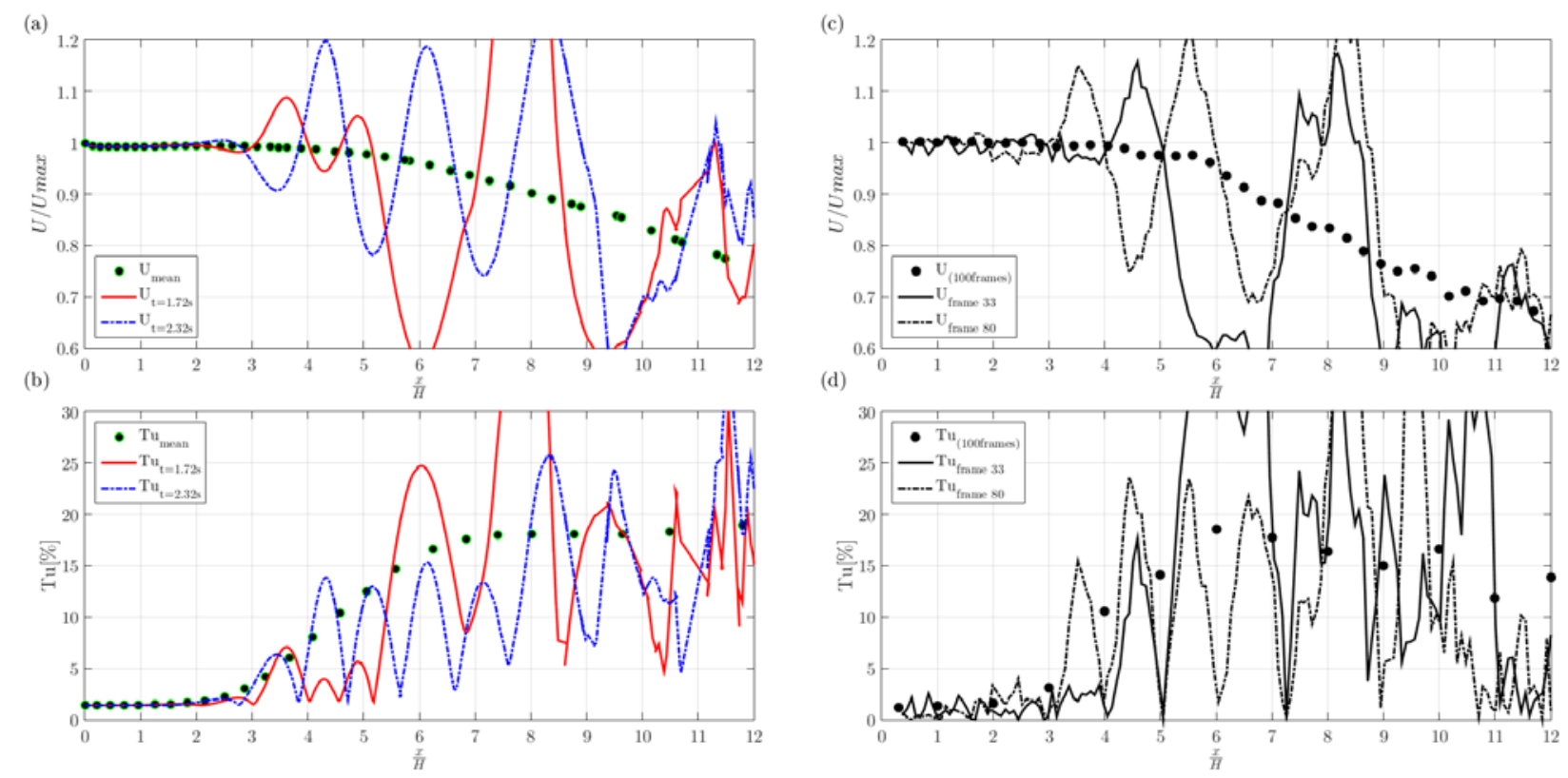

\section{Fig.11 - Average and instant velocity and turbulence along the centreline; numerical (colored, a,b) and experimental measurements (black-white, $c, d$ ) for $R e=6800$.}

The instant velocity profiles, predicted numerically at several distances from the slot exit before the PCR, are reported in Fig. 12 with circles and stars, respectively at $\mathrm{t}=1.72 \mathrm{~s}$ and $\mathrm{t}=2.32 \mathrm{~s}$, and compared to the instant measurements of [12], reported with dotted and continuous lines, and relative to the frames 33 and 80. The distances from the slot exit where the comparisons are carried on, reported in Fig. 12, are also indicated in Fig. 10 with vertical dotted lines.

On the exit of the jet, $\mathrm{x} / \mathrm{H}=0.38$, Fig. 12a, the two numerical predictions are in very good agreement with the measurements, as well as at $\mathrm{x} / \mathrm{H}=3.06$, Fig. 12b, where the flow is between the NDF and the SDF. At the distance of $x / H=3.59$, Fig. 12c, the velocity around the centerline of the jet, at $t=2.32 \mathrm{~s}$, frame 80 , is greater than at $\mathrm{t}=1.72 \mathrm{~s}$, frame 33 , because of the jet contraction, shown by Fig. 10b and d, in comparison to the jet height of Fig. 10a, 10c. This confirms that the jet is in the SDF, where the jet height has contraction/enlargement with consequent increase/decrease of the velocity.

At the distance of $\mathrm{x} / \mathrm{H}=4.44$, Fig. $12 \mathrm{~d}$, the flow is in the coherent vortices region. The velocity around the centerline is smaller at $\mathrm{t}=2.32 \mathrm{~s}$, frame 80 , than at $\mathrm{t}=1.72$, frame 33 , because the jet has an enlargement, evidenced by Fig. 10b, 10d, in contrast to the contraction shown by Fig. 10a, 10c. At the distance of $\mathrm{x} / \mathrm{H}=5.35$, Fig. $12 \mathrm{e}$, the centerline velocity is smaller at $\mathrm{t}=1.72 \mathrm{~s}$, frame 33 , than at $\mathrm{t}=2.32$, frame 80, because the jet height has an enlargement, evidenced by Fig. 10a, 10c, in contrast to the contraction shown by Fig. 10b, 10d. 

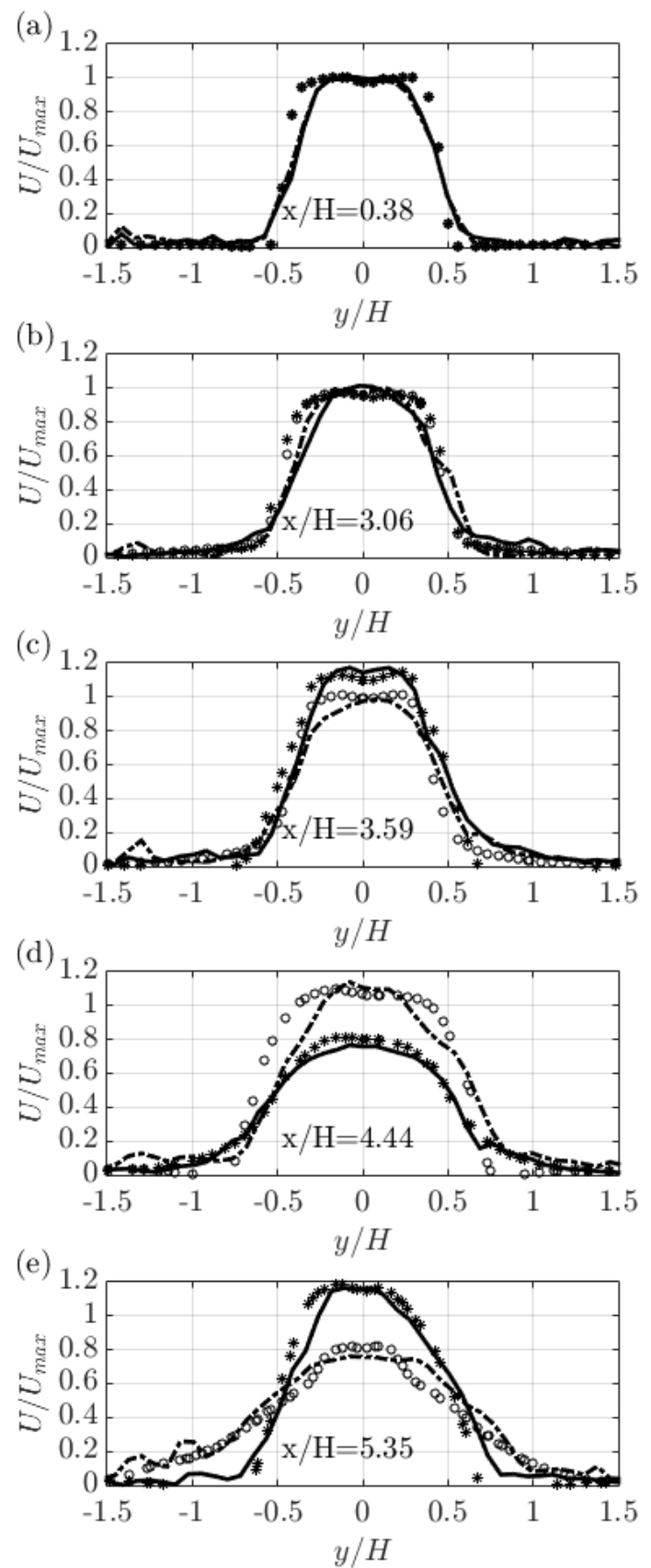

Figure 12 - Instant velocity profiles within the PCR for $\operatorname{Re}=6800$. Numerical $\left(\circ t=1.72 \mathrm{~s}, *_{t=2.32} \mathrm{~s}\right)$ and $P I V$ measurements (dotted line, frame 33; continuous line, frame 80).

$3.3-2 D, R e=10,400$.

Figure 13 presents the average concentration field (colored) obtained with the $2 \mathrm{D}$ numerical simulations at $\operatorname{Re}=10,400$. The height of the jet remains constant, and equal to the slot one, for a length equal to $\mathrm{x} / \mathrm{H}=2$, which identifies the length of the URF in the average numerical simulations. After the URF the darker concentration field decreases in thickness, identifying visually the Potential Core region, PCR, which ends around $\mathrm{x} / \mathrm{H}=5-6$. Both the lengths of URF and PCR agree with the experimental results of [10]. 
The numerical results of the concentration field are compared to the PIV average visualization, obtained for the same Reynolds number in [10], and reported on the same Fig. 13 with its envelope as dotted line. It is evident the good agreement between the 2D numerical simulations and the PIV average visualization envelope. The reduced jet expansion in the FDR for the numerical results can be due to the lack of vortex-breakdown.

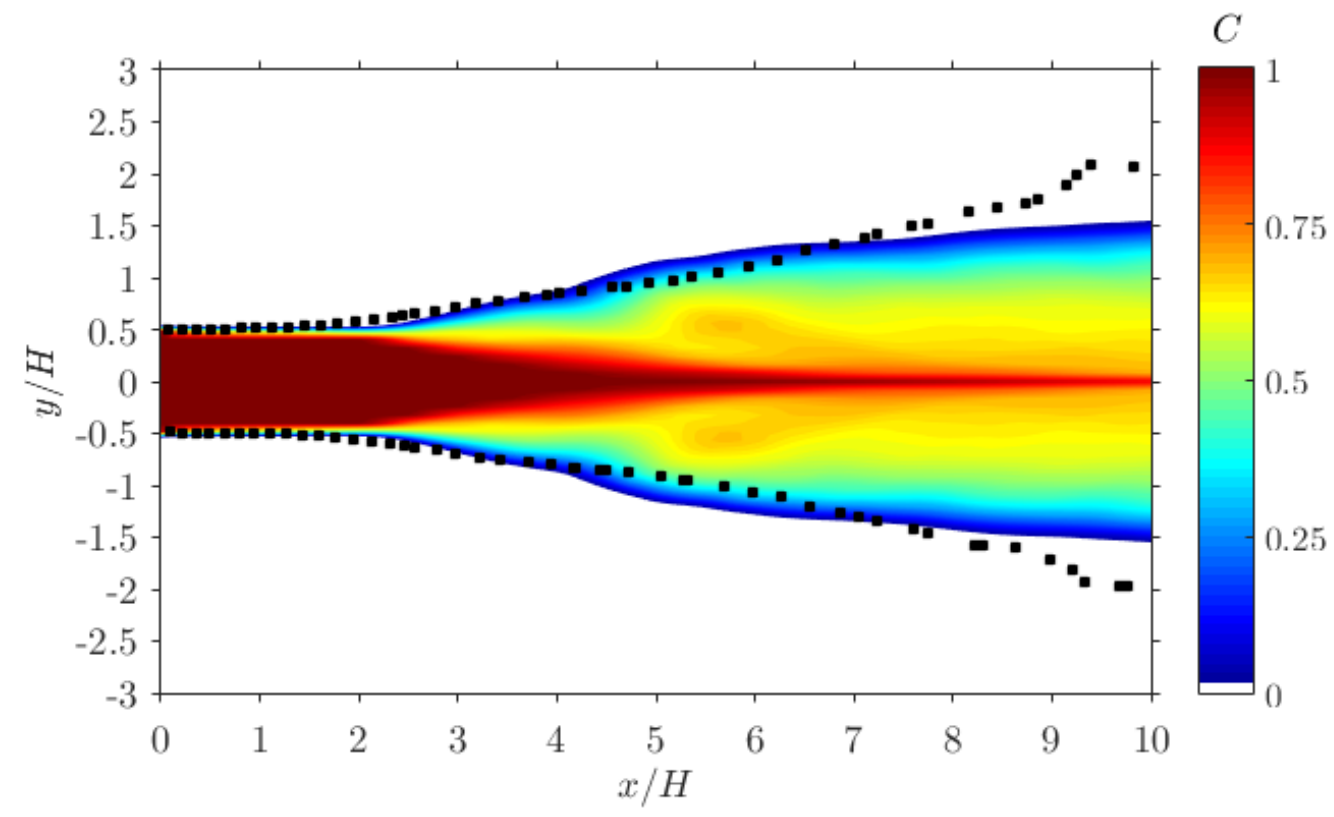

Fig. 13 - Average concentration field: 2D numerical (colored) and PIV envelope (dashed line) for Re=10,400.

The average velocity field at $\mathrm{Re}=10,400$, obtained with the $2 \mathrm{D}$ numerical simulation, is shown in Fig. 14a. The height of the jet remains almost constant, for a length equal to $\mathrm{x} / \mathrm{H}=2$, which identifies the length of the URF in the average numerical simulations. The darker triangular region, present after the URF and identifying visually the Potential Core region, PCR, ends around $\mathrm{x} / \mathrm{H}=5-6$. Both the lengths of URF and PCR agree with the experimental results of [10]. The 2D numerical results of the average velocity can be compared to the PIV measurements, obtained for the same Reynolds number, [10], and reported in Fig. 14b. Also for this Reynolds number it is evident the good agreement between the 2D numerical simulations and the PIV results.

The reduced jet expansion of the velocity, as well as of the concentration, in the numerical results of the FDR can be due to the lack of vortex-breakdown. 
(a)

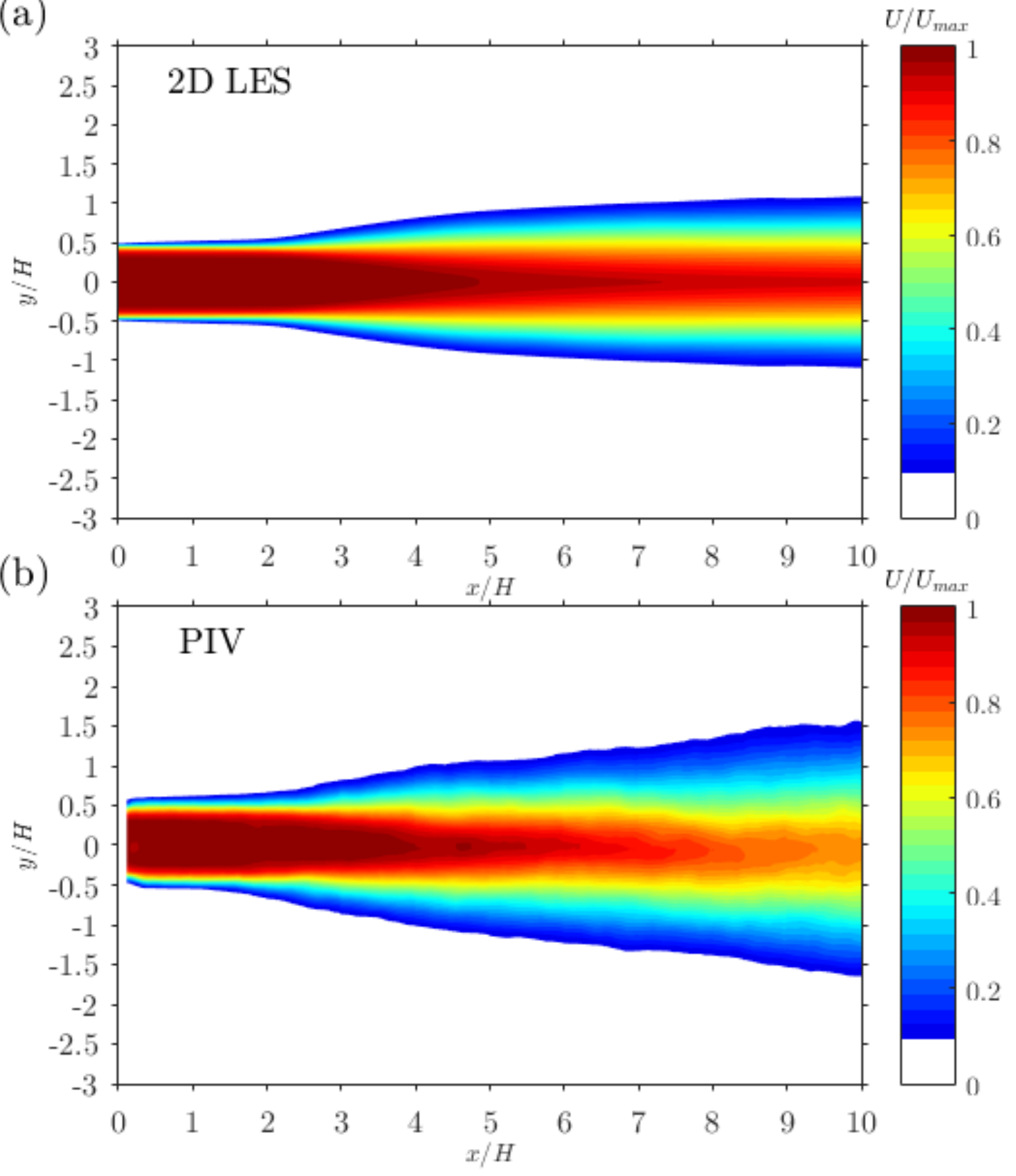

Fig. 14 - Average velocity field: numerical (a) and experimental PIV (b) results at $R e=10400$.

The average velocity profiles, predicted numerically at $\mathrm{Re}=10,400$ and presented in Fig. 15 from the slot exit up the end of the PCR with dots, are in reasonably good agreement to the average velocity measurements, reported with circles for PIV, and with star for HFA, [10]. The average turbulent velocity profile on the exit of the slot, given by Eqs. 15-16, is reported in Fig. 15a with a continuous line, in agreement to the experimental turbulent velocity profile measured in the experiments [10]. 

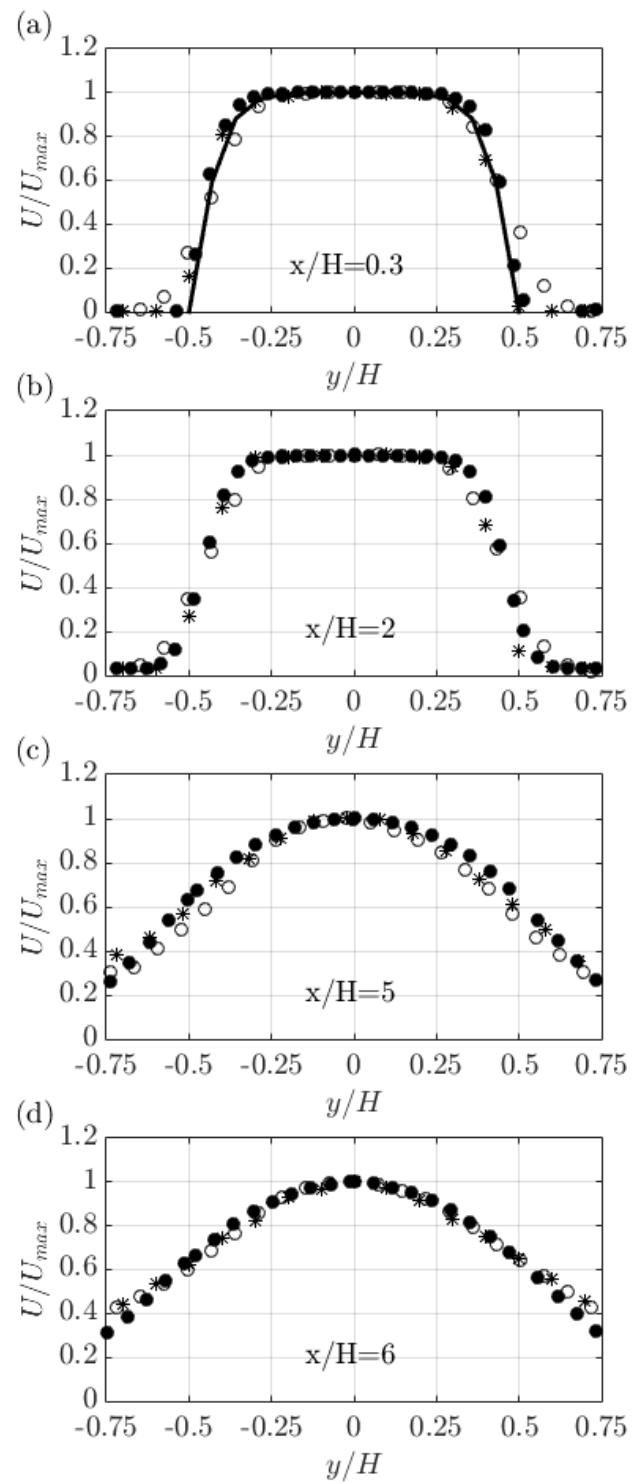

Figure 15 - Average velocity profiles: numerical (•), PIV (०) and HFA (*) measurements at Re=10,400.

The instant concentration fields, obtained with the 2D numerical simulations at $\mathrm{Re}=10,400$, are reported, colored, in Fig. 16 at two different instants, $t=0.8 \mathrm{~s}$ in Fig. 13a, and $\mathrm{t}=0.82 \mathrm{~s}$ in Fig. 16b. They are in qualitative agreement to the experimental instant concentration PIV images, black and white, and identified by the frames 01,11 , obtained in the experiments [12] and never published before.

Figures 16a and $16 \mathrm{~b}$ show a NDF long about $\mathrm{x} / \mathrm{H}=2$, followed by the SDF, up to $\mathrm{x} / \mathrm{H}=3$, where the height increases, here symmetrically, without the formation of vortices. Both the lengths of NDF and SDF are in agreement with the experimental measurements of [12]. Figure 16a shows, after the SDF, a pair of symmetric vortices, as the PIV image in Fig. 16c, frame 01, typical of the turbulent flow. The pair of vortices is followed by two big vortices, with a qualitative good agreement between numerical simulations and experimental data up to the vortex breakdown, which is not predictable with the 2D approach. 
Figure 16b shows, after the SDF, two pairs of symmetric vortices, as the PIV image of Fig. 16d, frame 11, typical of the turbulent flow. The two pairs of vortices are followed by one big vortex, with a qualitative good agreement between numerical simulations and experimental data up to the vortex breakdown, which is not predictable with the 2D approach.
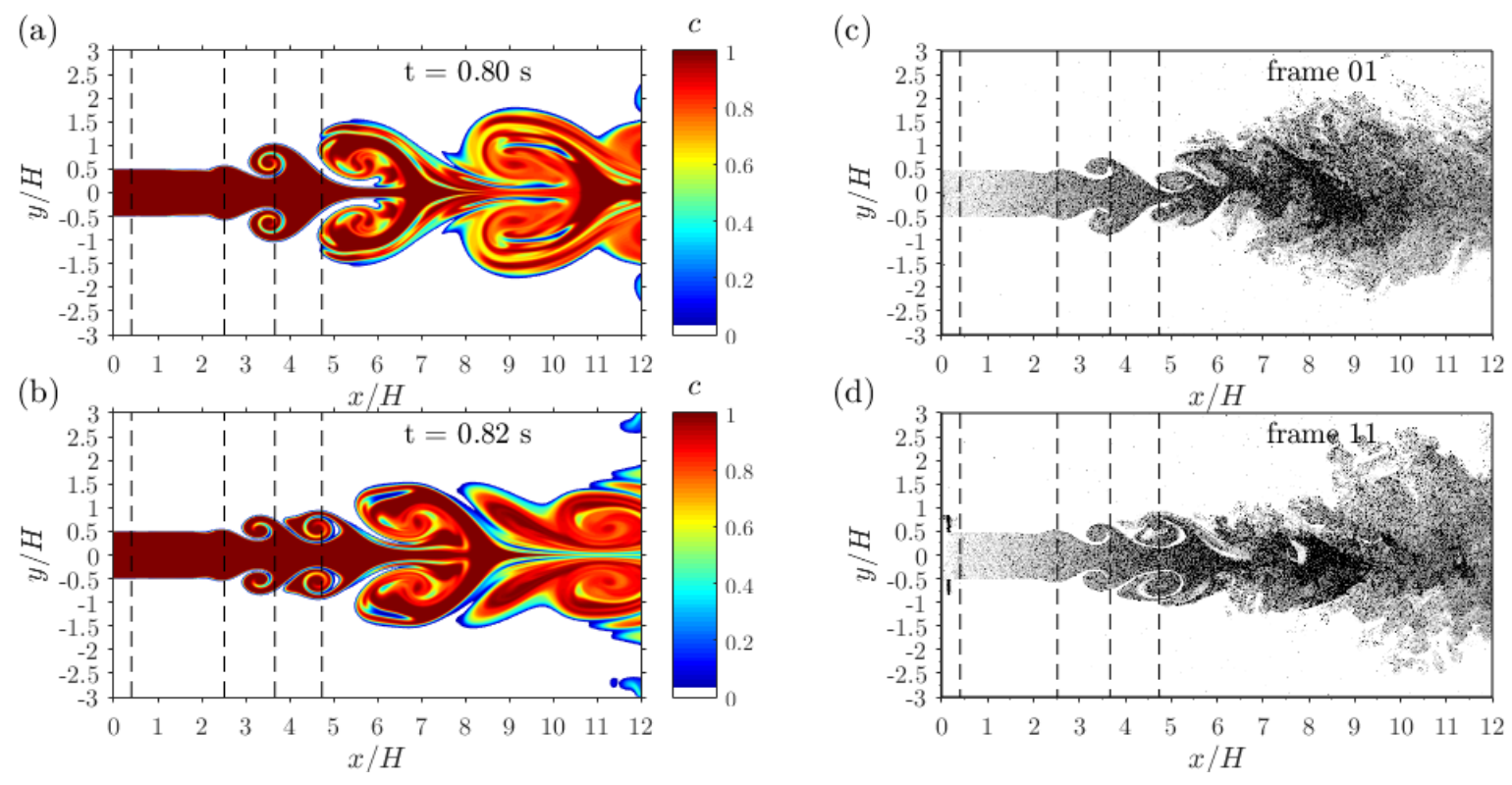

Fig. 16 - Instant concentration field: numerical (colored, a,b) and experimental PIV (black-white, c,d) results at $\operatorname{Re}=10,400$.

The instant velocity profiles, predicted numerically at several distances from the slot exit, are reported in Fig. 17 with circles and stars, respectively after $\mathrm{t}=0.80 \mathrm{~s}$ and $\mathrm{t}=0.82 \mathrm{~s}$, and compared to the instant measurements, [12], reported with dotted and continuous lines, and relative to the frames 01 and 11 . The distances from the slot exit where the comparisons are carried on are also indicated in Fig. 16 with vertical dotted lines.

On the exit of the jet, at $\mathrm{x} / \mathrm{H}=0.4$, Fig. 17a, the two numerical predictions are in good agreement with the measurements, as well as at $\mathrm{x} / \mathrm{H}=2.52$, Fig. $17 \mathrm{~b}$, where the flow is within the SDF. At the distance of $\mathrm{x} / \mathrm{H}=3.67$, Fig. 17c, the velocity around the centerline of the jet, at $\mathrm{t}=0.80 \mathrm{~s}$, frame 01 , is greater than at $\mathrm{t}=0.82 \mathrm{~s}$, frame 11, because of the jet contraction, shown by Fig. 16a, in comparison to the jet height of Fig. 16b. At $\mathrm{x} / \mathrm{H}=4.74$, Fig. $17 \mathrm{~d}$, the velocity around the centerline of the jet is greater at $\mathrm{t}=0.80 \mathrm{~s}$, frame 01 , than at $\mathrm{t}=0.82 \mathrm{~s}$, frame 11, because of the jet contraction, shown by Fig. 16a, as before. 

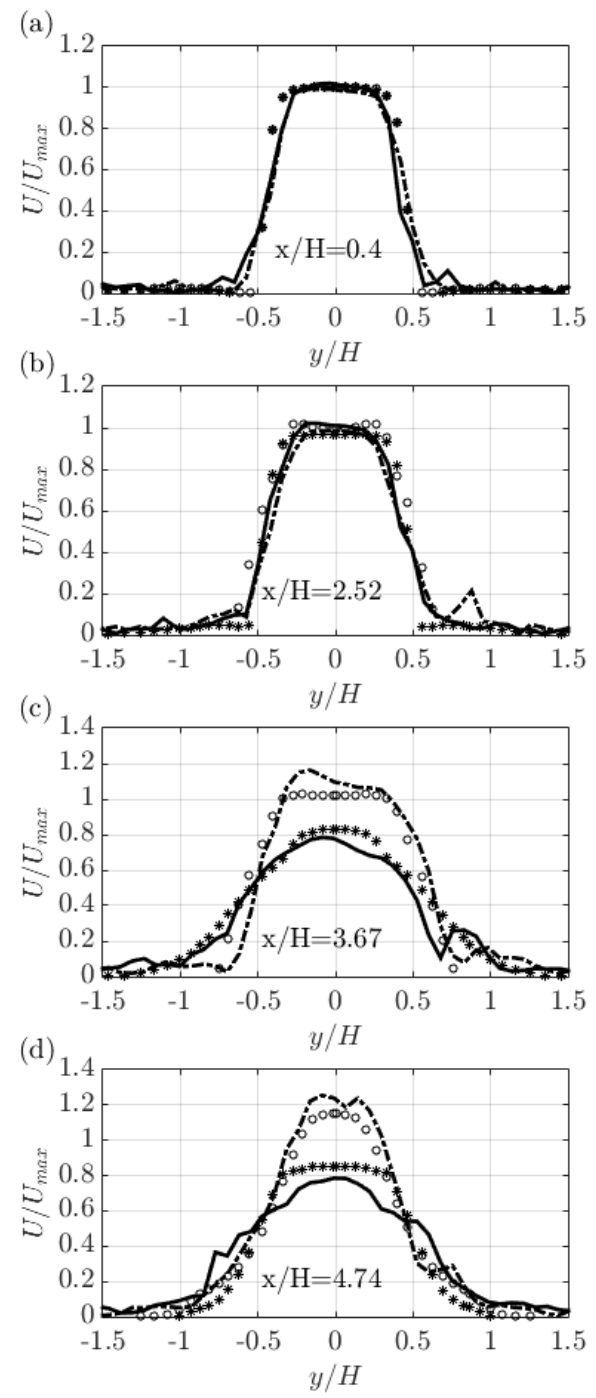

Figure 17 - Instant velocity profiles at $R e=10,400$. Numerical ( $\circ t=0.80 \mathrm{~s},{ }^{*} t=0.82 \mathrm{~s}$ ) and PIV measurements (dotted line, frame 01; continuous line, frame 11).

$3.4-2 D$, Reynolds $=22,000$.

Figure 18 presents the average concentration field (colored) obtained with the 2D numerical simulations at $\operatorname{Re}=22,000$. The height of the jet remains constant, and equal to the slot one, for a length equal to $\mathrm{x} / \mathrm{H}=1-2$, which identifies the length of the URF in the average numerical simulations. After the URF the darker concentration field decreases in thickness, identifying visually the Potential Core region, PCR, which ends around $\mathrm{x} / \mathrm{H}=5$. Both the lengths of URF and PCR agree with the experimental results of [10].

The numerical results of the concentration field are compared to the PIV average visualization, obtained for the same Reynolds number in [10], and reported on the same Fig. 18 with its envelope as dotted line. It is evident the good agreement between the 2D numerical simulations and the PIV average visualization envelope. The reduced jet expansion in the FDR for the numerical results can be due to the lack of vortex-breakdown. 


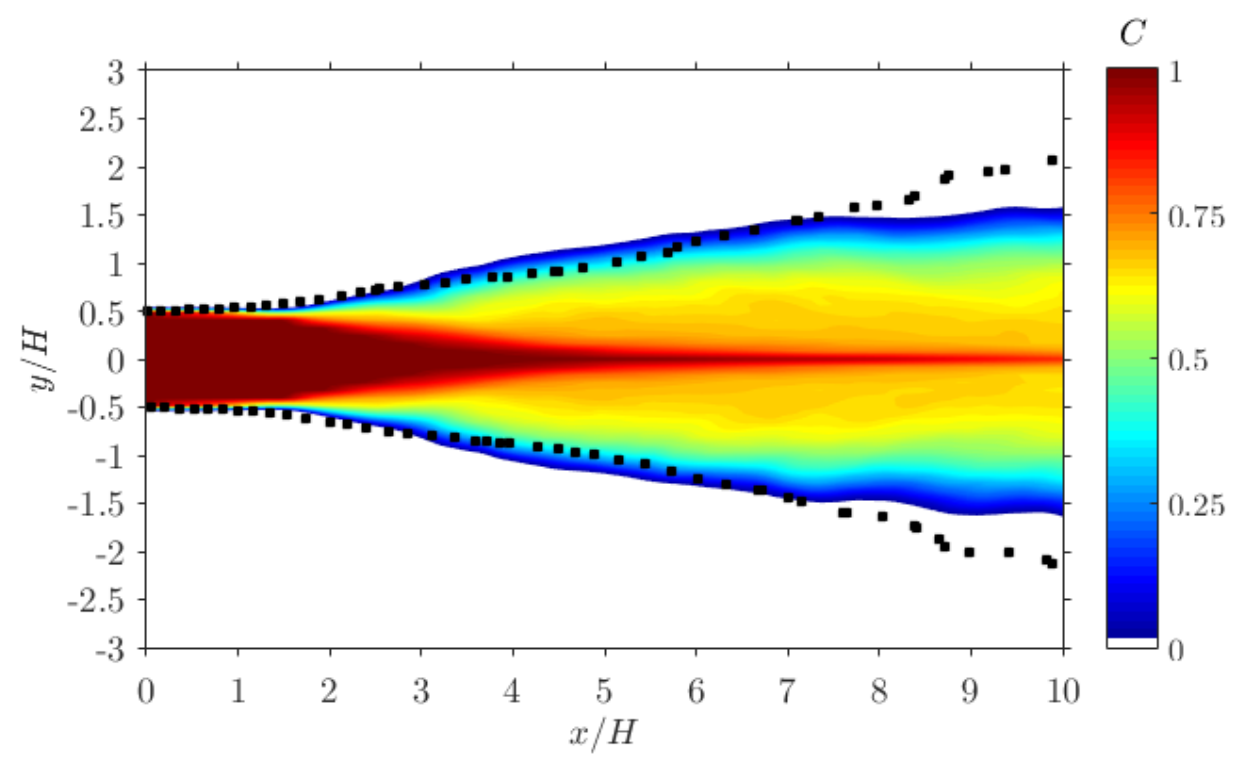

Fig. 18 - Average concentration field: 2D numerical (colored) and PIV envelope (dashed line) for $R e=22,000$.

The average velocity field, obtained with the $2 \mathrm{D}$ numerical simulation at $\mathrm{Re}=22,000$, is shown in Fig. 19a. The height of the jet remains almost constant, for a length equal to $\mathrm{x} / \mathrm{H}=1-2$, which identifies the length of the URF in the average numerical simulations. The darker triangular region, present after the URF and identifying visually the Potential Core region, PCR, ends around $\mathrm{x} / \mathrm{H}=4-5$. Both the lengths of URF and PCR agree with the experimental results of [10]. The 2D numerical results of the average velocity can be compared to the PIV measurements, obtained for the same Reynolds number, [10], and reported in Fig. 19b. Also for this Reynolds number it is evident the good agreement between the 2D numerical simulations and the PIV results.

The reduced jet expansion of the velocity, as well as of the concentration, in the numerical results of the FDR can be due to the lack of vortex-breakdown. 
(a)

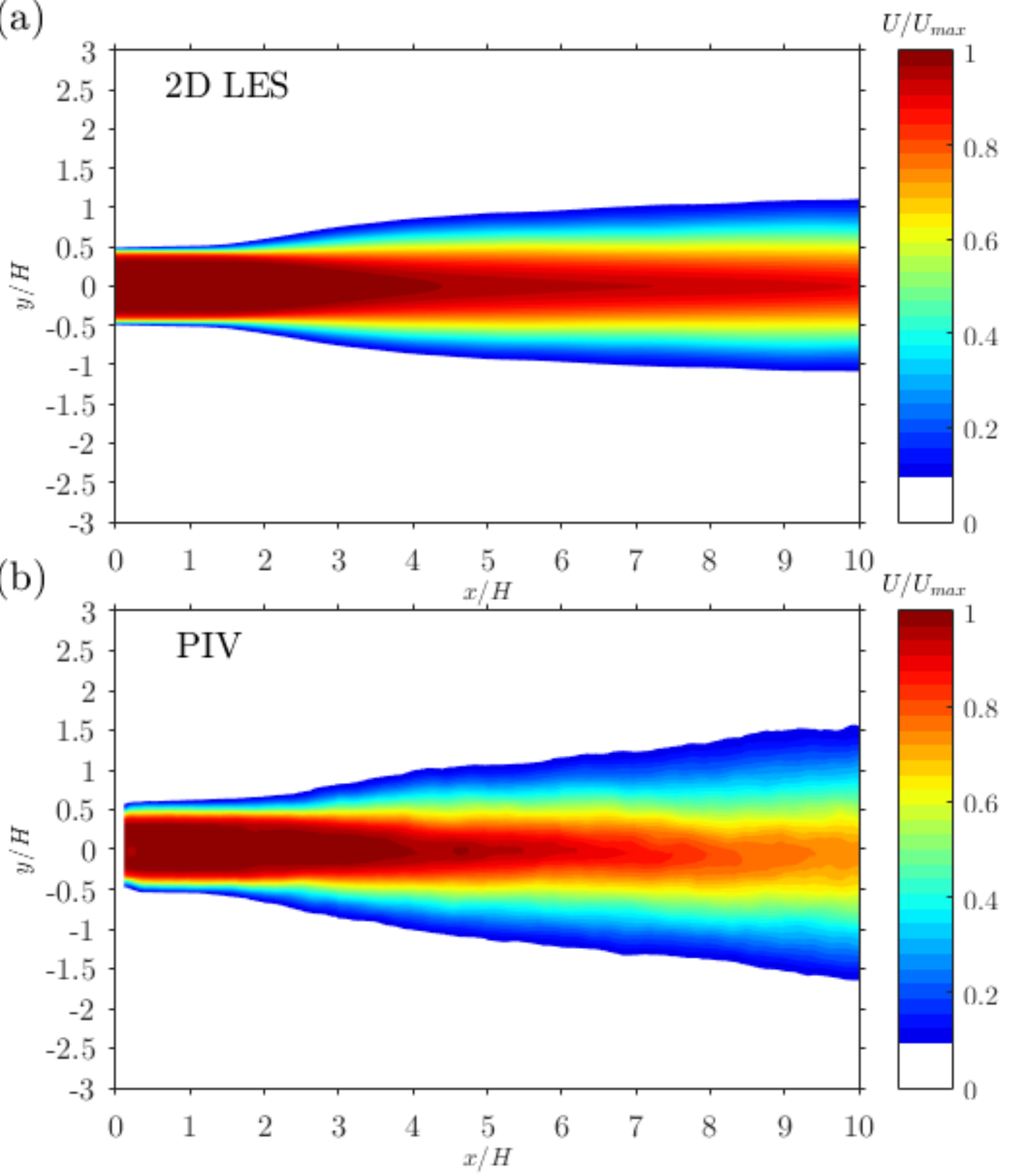

Fig. 19 - Average velocity field: numerical (a) and experimental PIV (b) results at $R e=22000$.

The average velocity profiles, predicted numerically at $\mathrm{Re}=22,000$, and presented in Fig. 20 from the slot exit up the end of the PCR with dots, are in reasonably good agreement to the average velocity measurements, reported with circles for PIV, and with star for HFA, [10]. 
The average turbulent velocity profile on the exit of the slot, given by Eqs. 15-16, is reported in Fig. 20a with a continuous line, in agreement to the experimental turbulent velocity profile measured in the experiments [10].
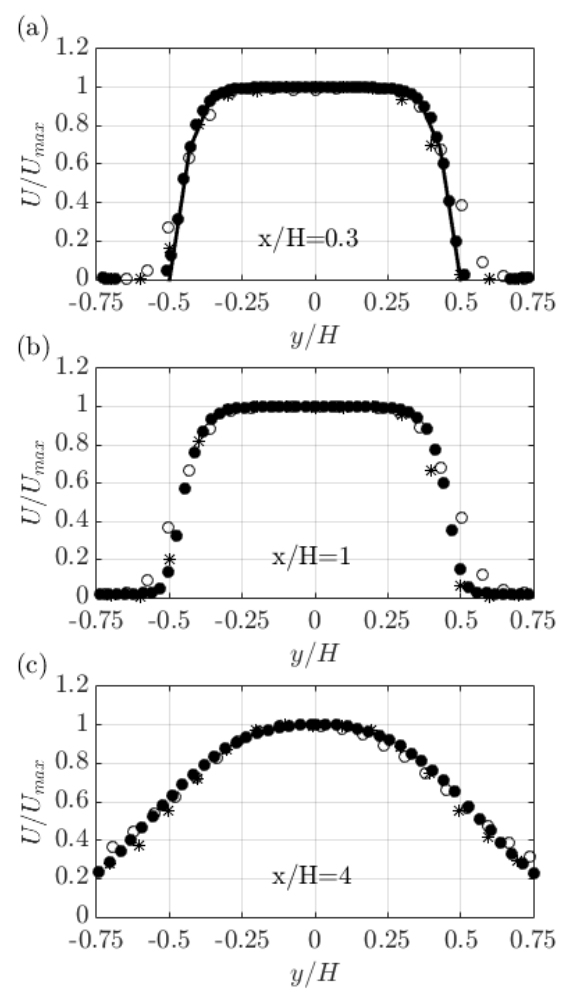

Figure 20 - Average velocity profiles: numerical (•), PIV (०) and HFA (*) measurements at Re=22,000.

The instant concentration fields, obtained with the 2D numerical simulations at $\mathrm{Re}=22,000$, are reported, colored, in Fig. 21 at two different instants, t=0.55 s in Fig. 21a, and t=0.554 s in Fig. 21b. They are in qualitative agreement to the experimental instant concentration PIV images, black and white, identified by the frames 05,08 , and obtained in the experiments [12], but never published before.

Figures 21a and 21b show a NDF long about $\mathrm{x} / \mathrm{H}=1-2$, followed by the SDF, up to $\mathrm{x} / \mathrm{H}=2$, where the height increases, here symmetrically, without the formation of vortices. Both the lengths of NDF and SDF are in agreement with the experimental measurements of [12]. Figures 21a and 21b show, after the SDF, a pair of symmetric vortices, as the PIV images in Fig. 21c, frame 05, and Fig. 21d, frame 08, typical of the turbulent flow. The pair of vortices is followed by two big vortices, with a qualitative good agreement between numerical simulations and experimental data up to the vortex breakdown, which is not predictable with the 2D approach. 


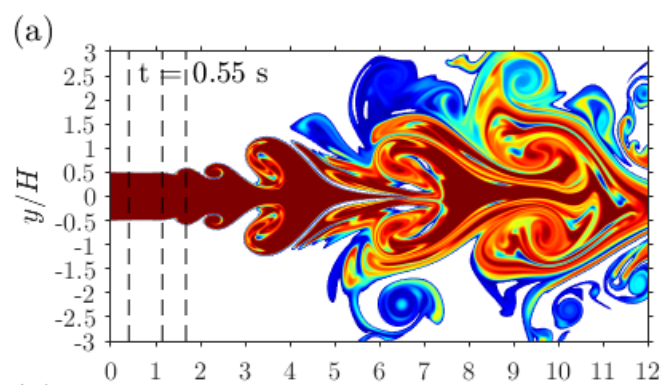

(b)

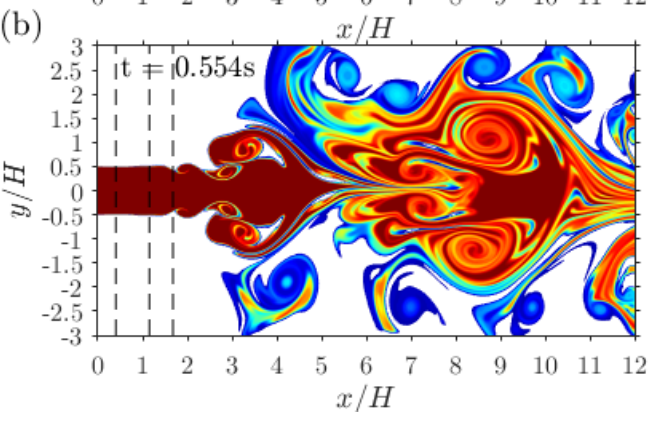

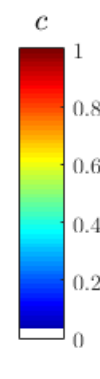

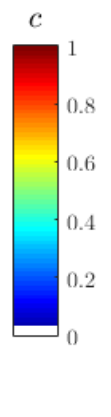

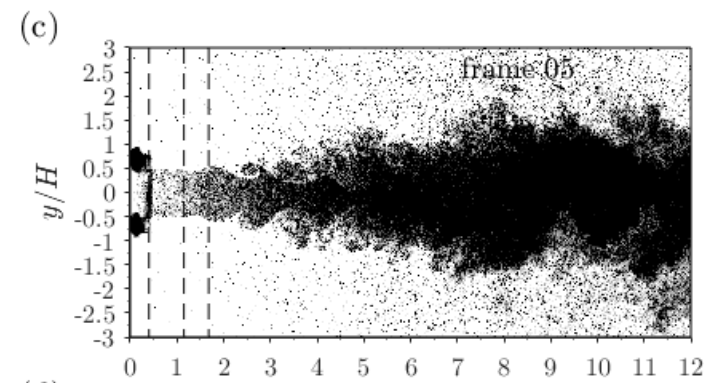

(d)

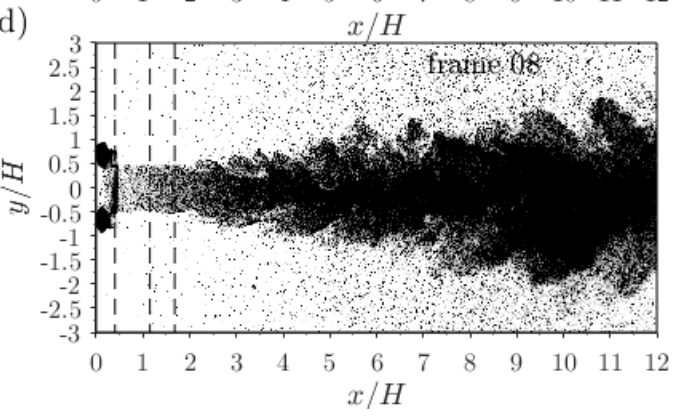

Fig. 21 - Instant concentration field: numerical (colored, a, b) and experimental PIV (black-white, c,d) results at $R e=22,000$.

The instant velocity profiles, predicted numerically at several distances from the slot exit, are reported in Fig. 22 with circles and stars, respectively after $\mathrm{t}=0.55 \mathrm{~s}$ and $\mathrm{t}=0.554 \mathrm{~s}$, and compared to the instant measurements, [12], reported with dotted and continuous lines, and relative to the frames 05 and 08. The distances from the slot exit where the comparisons are carried on are also indicated in Fig. 21 with vertical dotted lines.

On the exit of the jet, at $\mathrm{x} / \mathrm{H}=0.4$, Fig. 22a, the two numerical predictions are in good agreement with the measurements, as well as at $\mathrm{x} / \mathrm{H}=1.15$, Fig. 22b, where the flow is still within the NDF. At the distance of $\mathrm{x} / \mathrm{H}=1.68$, in the SDF, Fig. 22c, the velocity around the centerline of the jet at $\mathrm{t}=0.554 \mathrm{~s}$, frame 08 , is greater than at $t=0.55 \mathrm{~s}$, frame 05, because of the jet contraction, shown by Fig. 21b, in comparison to the jet height of Fig. 21a, frame 05. 

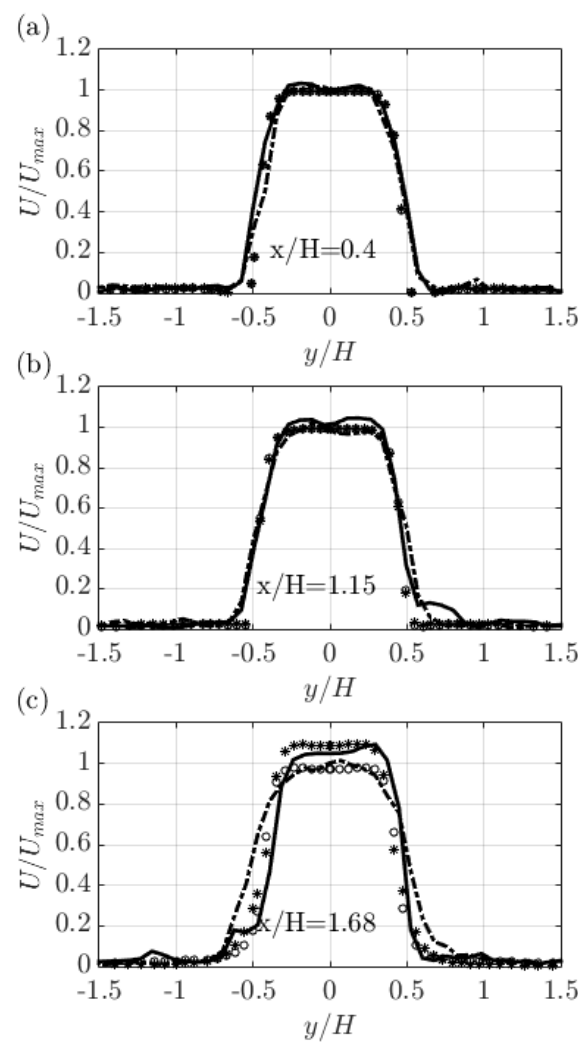

Figure 22 - Instant velocity profiles at $R e=22,000$. Numerical $(\circ t=0.55 \mathrm{~s}, * t=0.554 \mathrm{~s})$ and PIV measurements (dotted line, frame 05; continuous line, frame 08).

\section{Conclusions}

A series of 2-3 D Large Eddy Simulations (LES) of the mass transfer and flow evolution of rectangular free jets are carried out at four Reynolds numbers in the range from 3400 to 22,000, i.e. $3400,6800,10,400$ and 22,000. The numerical concentration and velocity fields and the fluid dynamics variables are compared with the experimental counterparts, measured with the Particle Image Velocimetry (PIV) and the Hot Film Anemometry (HFA). The numerical simulations assume the Schmidt number equal to 100, because of the dimensions of the particles employed in the PIV experiments, while the turbulence assumed on the exit of the slot is equal to $1.5-2 \%$, as measured experimentally.

The numerical approach is 3D at the two smallest Reynolds numbers, while 2D at the two greatest Reynolds to save computational times costs. The 3D approach is capable of reproducing the near field region and the fully developed region with the vortex breakdown. The 2D numerical simulations cannot predict the vortex breakdown but they are able to reproduce the mass transfer with the concentration field, and the flow evolution in the near field region, NFR, before the fully developed region, FDR.

The average concentration and velocity fields, at the four Reynolds numbers, compare favorably the PIV images, showing the presence of the URF, undisturbed region of flow, with a good agreement with the experimental lengths, which decrease with the increase of the Reynolds number. The average concentration 
and velocity fields show also the potential core regions, PCR, in good agreement with the experimental lengths. The numerical average velocity profiles, at several distances from the slot exit within the PCR, are in good agreement to the experimental measurements.

The instant numerical concentration fields, taken at several instants of time, compare successfully the corresponding PIV images, with a good agreement of the general pictures. The numerical instant concentration fields confirm the presence of the negligible disturbances flow, NDF, where the jet height maintains constant and equal to the slot one, and the small disturbances flow, SDF, where the jet height enlarges or/and contracts slightly without the formation of vortices. The lengths of NDF and SDF are also in good agreement to the experimental ones. The numerical instant velocity profiles, at several distances from the slot exit within the PCR and different instant of times, compare favorably the experimental ones showing their increase or decrease according to the relative variation of the flow section of the jet, in agreement to the experimental measurements.

Average and instant velocity and turbulence numerical results on the centreline, with their fluctuations, are in good agreement to the experiments.

\section{References}

[1] M. L. Albertson, Y. B. Dai, R. A. Jensen, and H. Rouse, Diffusion of Submerged Jets, Trans. Am. Soc. Civ. Eng., 410 (115) 1 (1950) 639-64.

[2] B. G. Van der Hegge Zijnen, Measurements of the Distribution of Heat and Matter in a Plane Turbulent Jet of Air, Appl. Sci. Res., 7 (4) (1958) 277-292.

[3] L. J. S. Bradbury, The Structure of a Self-Preserving Turbulent Plane Jet, Journal of Fluid Mechanics, 23 (1) (1965) 31-64.

[4] E. Gutmark, and I. Wygnanski, The Planar Turbulent Jet, Journal of Fluid Mechanics, 73(3) (1976) 465.

[5] G.N. Abramovich, On the Deformation of the Rectangular Turbulent Jet Cross-Section, International Journal of Heat and Mass Transfer, 25 (12) (1982) 1885-1894.

[6] F. Gori, F. De Nigris, E. Nino, Fluid Dynamics Measurements and Optical Visualization of the Evolution of a Submerged Slot Jet of Air, Proceedings of the 12th International Heat Transfer, Editors: J. Taine, Editions Elsevier, Grenoble, France, 2 (2002) 303-308.

[7] F. Gori, I. Petracci, Fluid Dynamics Measurements in a Submerged Free Slot Jet of Air, Proceedings of the 14th International Symposium on Transport Phenomena, Bali Indonesia, 6-10th July, (2003) 491-496. 
[8] F. Gori, E. Nino, Fluid Dynamics Measurements and Flow Visualizations of a Free Slot Jet of Air, American Society of Mechanical Engineers, Fluids Engineering Division (Publication) FED, 259 (2003) 187-192.

[9] F. Gori, E. Nino, I. Petracci, Shadowgraph Visualizations of a Submerged Free Slot Jet of Air, International Journal of Heat and Technology, 25 (1) (2007) 157-164.

[10] F. Gori, I. Petracci, M. Angelino, Flow Evolution of a Turbulent Submerged Two-Dimensional Rectangular Free Jet of Air. Average Particle Image Velocimetry (PIV) Visualizations and Measurements, International Journal of Heat and Fluid Flow, 44 (2013) 764-775.

[11] F. Gori, I. Petracci, Fluid Dynamics Measurements and Numerical Simulations around a Circular Cylinder Impinged by a Submerged Slot Jet of Air, American Society of Mechanical Engineers, Fluids Engineering Division (Publication) FED, 259 (2003) 179-185.

[12] F. Gori, I. Petracci, M. Angelino, Influence of the Reynolds number on the instant flow evolution of a turbulent rectangular free jet of air, International Journal of Heat and Fluid Flow, 50 (2014) 386-401.

[13] W. Tollmien, Calculation of Turbulent Expansion Processes, Zeitschrift Für Angewandte Mathematik Und Mechanik, 6 (6) (1926) 468-78.

[14] H. Gortler, Berechnung von Aufgaben Der Freien Turbulenz Auf Grund Eines Neuen Naherungsansatzes, Zeitschrift Für Angewandte Mathematik Und Mechanik, 22 (5) (1942) 244-254.

[15] E. Forthmann, Turbulent Jet Expansion, NACA-TM-789, 1936.

[16] D.R. Miller and E.W. Comings, Static Pressure Distribution in the Free Turbulent Jet, J. Fluid Mech., 3 (1) (1957) 1-16.

[17] H. W. Liepmann and J. Laufer, Investigations of Free Turbulent Mixing, NACA Technical Note, 1947.

[18] F. O. Thomas and V. W. Goldschmidt, Structural Characteristics of a Developing Turbulent Planar Jet, J. Fluid Mech., 163 (1986) 227-256.

[19] L. J. S. Bradbury, The Structure of a Self-Preserving Turbulent Plane Jet, Journal of Fluid Mechanics 23 (1) (1965) 31-64.

[20] A. A. Sfeir, The Velocity and Temperature Fields of Rectangular Jets, International Journal of Heat and Mass Transfer 19 (11) (1976) 1289-97.

[21] A. Krothapalli, D. Baganoff, and K. Karamcheti, On the Mixing of a Rectangular Jet, Journal of Fluid Mechanics 107 (1981): 201-20. 
[22] F. Gori, L. Bossi, On the Cooling Effect of an Air Jet along the Surface of a Cylinder, International Communications in Heat and Mass Transfer, 27 (5) (2000) 667-676.

[23] F. Gori, L. Bossi, Optimal Slot Height in the Jet Cooling of a Circular Cylinder, Applied Thermal Engineering, 23 (7) (2003) 859-870.

[24] F. Gori, I. Petracci, Influence of screen solidity ratio on heat transfer upon a cylinder impinged by a rectangular jet, International Journal of Heat and Mass Transfer, 81 (2015) 19-27.

[25] F. Gori, G. Pacchiarotti, I. Petracci, Pulsating Jets Cooling Circular Cylinders, American Society of Mechanical Engineers, Process Industries Division (Publication) PID, 8 (2003) 153-159.

[26] F. Gori, I. Petracci, M. Angelino, Experimental and Numerical Heat Transfer on a Cylinder Cooled by Two Rectangular Jets of Different Heights, ASME International Mechanical Engineering Conference and Exposition, Proceedings (IMECE), 7 (D) (2012) 2679-2688.

[27] F. Gori, I. Petracci, Influence of turbulence on heat transfer upon a cylinder impinged by a slot jet of air, Applied Thermal Engineering, 49 (2012) 106-117.

[28] F. Gori, I. Petracci, On the Effect of the Slot Height in the Cooling of a Circular Cylinder with a Rectangular Je,. International Communications in Heat and Mass Transfer 48 (2013) 8-14.

[29] F. Gori, F. De Nigris, E. Pippione, G. Scavarda, Cooling of Finned Cylinders by a Jet Flow of Air, American Society of Mechanical Engineers, Process Industries Division (Publication), PID, 7 (2002) 117122.

[30] F. Gori, M. Borgia, A. Doro Altan, Cooling of a Finned Cylinder by a Jet Flow of Air, American Society of Mechanical Engineers, Process Industries Division (Publication) PID, 8 (2003) 147-152.

[31] F. Gori, M. Borgia, A. Doro Altan, M. Mascia, I. Petracci, Cooling of a Finned Cylinder by a Jet Flow of Air, ASME Journal of Heat Transfer, 127 (12) (2005) 1416-1421.

[32] F. Gori, M. Mascia, I. Petracci, Air cooling of a finned cylinder with slot jets of different height, International Journal of Thermal Sciences, 50 (2011) 1583-1593.

[33] I. Petracci, L. Manni, F. Gori, Numerical simulation of the optimal spacing for a radial finned tube cooled by a rectangular jet. I-Average thermal results, International Journal of Thermal Sciences, 104 (2016) 54-67.

[34] F. Gori, L. Bossi, Cooling of Two Cylinders in a Row by a Slot Jet of Air, International Journal of Transport Phenomena, 4 (2002) 245-256.

[35] F. Gori, I. Petracci, V. Tedesco, Cooling of two smooth cylinders in row by a slot jet of air with low turbulence, Applied Thermal Engineering, 27 (14-15) (2007) 2415-2425. 
[36] F. Gori, P. Coppa, Circumferential variation of heat transfer on three circular cylinders cooled by a slot jet of air, International Journal of Heat and Technology, 16 (2) (1998) 63-69.

[37] F. Gori, L. Bossi, On the optimal configuration of three cylinders in a row cooled by a slot jet of air, American Society of Mechanical Engineers. Heat Transfer Division (Publication), Fundamentals of singlephase convective heat transfer, HTD, 366 (2000) 39-44.

[38] F. Gori, I. Petracci, Heat Transfer Measurements and Numerical Simulations in the Cooling of a Circular Cylinder by a Slot Jet of Air, American Society of Mechanical Engineers, Heat Transfer Division, (Publication) HTD, 374 (1) (2003) 3-9.

[39] F. Gori, M. Angelino, A. Boghi, I. Petracci, Preliminary Numerical Solutions of the Evolution of Free Jets, ASME International Mechanical Engineering Conference and Exposition, Proceedings (IMECE), 7 (D) (2012) 463-469.

[40] A. Boghi, M. Angelino, F. Gori, Numerical evidence of an undisturbed region of flow in a turbulent rectangular submerged free jet, Numerical Heat Transfer, Part A: Applications, 70 (1) (2016) 14-29.

[41] M. Angelino, A. Boghi, F. Gori, Numerical solution of three-dimensional rectangular submerged jets with evidence of the undisturbed region of flow, Numerical Heat Transfer, Part A: Applications, 70 (8) (2016) 815-830.

[42] Andrea Boghi, Ivan Di Venuta, Fabio Gori, Passive Scalar Diffusion in the Near Field Region of Turbulent Rectangular Submerged Free Jets, International Journal of Heat and Mass Transfer, 112 (2017) 1017-1031.

[43] M.A. Islam, Einstein-Smoluchowski diffusion equation: a discussion, Physica Scripta, 70 (2-3) (2004) 120.

[44] E. Cunningham, On the velocity of steady fall of spherical particles through fluid medium, Proceedings of the Royal Society of London, Series A, Containing Papers of a Mathematical and Physical Character, 83 (563) (1910) 357-365.

[45] P. Moin, K. Squires, W. Cabot, and S. Lee, 'A dynamic sub-grid-scale model for compressible turbulence and scalar transport, Phys. Fluids 3 (1991) 2746.

[46] S. Lee, S. Lele, P. Moin, Simulation of spatially evolving compressible turbulence and the application of taylors hypothesis, Phys. Fluids A 4 (1992) 1521-1530. 